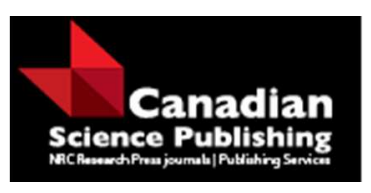

Canadian Journal of Physics

Revue canadienne de physique

\title{
Qualitative Study of Anisotropic Cosmological Models with Dark Sector Coupling
}

\begin{tabular}{|r|l|}
\hline Journal: & Canadian Journal of Physics \\
\hline Manuscript ID & cjp-2017-0088.R1 \\
\hline Manuscript Type: & Article \\
\hline Date Submitted by the Author: & $15-$ Mar-2017 \\
\hline Complete List of Authors: & $\begin{array}{l}\text { Chaubey, R.; Banaras Hindu University, DST - CIMS } \\
\text { Raushan, Rakesh; Banaras Hindu University Faculty of Science, DST-CIMS }\end{array}$ \\
\hline $\begin{array}{r}\text { Keyword: } \\
\text { Is the invited manuscript for } \\
\text { consideration in a Special } \\
\text { Issue? : }\end{array}$ & $\begin{array}{l}\text { LRS Bianchi type-I Universe, Dynamical Systems, Dark Energy, Coupling } \\
\text { parameter, Stability }\end{array}$ \\
\hline &
\end{tabular}




\title{
Qualitative Study of Anisotropic Cosmological Models with Dark Sector Coupling
}

\author{
R. Chaubey*and Rakesh Raushan ${ }^{\dagger}$ \\ Centre for Interdisciplinary Mathematical Sciences \\ Institute of Science \\ Banaras Hindu University \\ Varanasi, India
}

\begin{abstract}
In this paper, we study the dynamical evolution of LRS Bianchi type I cosmological model with coupling of dark sector. We investigate the phaseplane analysis when dark energy is modelled as exponential quintessence, and is coupled to dark energy matter via linear coupling between both dark components. The evolution of cosmological solutions is studied by using dynamical systems techniques. Stability and viability issues for three different physically viable linear coupling between both dark components are presented and discussed in detail.
\end{abstract}

Keywords: LRS Bianchi type-I Universe, Dynamical Systems, Dark Energy. PACS Nos: $98.80 \mathrm{Cq}$, 04.20.-q

*yahoo_raghav@rediffmail.com, rchaubey@bhu.ac.in

†rakeshraushanjha@gmail.com 


\section{Introduction}

The recent evidences of SNe Ia [1, 2, 3, 4, 5, 6, 7, 8], CMBR [9, 10], LSS [11, 12], SDSS [13, 14], WMAP [15] and Chandra X-ray observatory [16] have suggested that the universe is undergoing to accelerated expansion. Recent observations suggested that the dark energy is responsible for driving the late-time acceleration of the universe. Dark energy has never been detected or created in laboratory, therefore the idea of dark energy is hypothetical. The recent Hubble's diagram suggested that the more than $71 \%$ of the energy of the universe is in the form of dark energy and dark matter is believed to about $24 \%$ of the universe. One of the simplest scalar field models of time-evolving dark energy is quintessence. Without violating the observational constraints, dark energy may interact with dark matter by means of energy transfer between each other. In the past history of the universe, structure formation is one of the example of the interaction between dark components of the universe. In the several works, $[11,17,18,19,20,21,22$, $23,24]$ authors have discussed the various models of dark energy with interacting dark fluid. Recently some authors [25, 26, 27, 28, 29] have suggested that the energy density of dark energy and dark matter is of the same order and there is a possibility of coupling between them. An expression for general interaction between a scalar field $\phi$ (that contains dark energy) and dark matter is defined as the covariant derivative of the energy momentum tensor of the scalar field $\phi$ and covariant derivative of non-relativistic matter with opposite sign of interaction term.

Some authors $[30,31,32,33,34,35,36,37,38,39,40,41]$ have stressed that the Bianchi universes provide a generalization of the Friedmann-Lemaitre model (based on homogeneous and isotropic Robertson-Walker model). Therefore, Bianchi universe have played an important role in observational cosmologyl. Some authors [42, 43, 44] have used dynamical systems methods to study the evolution of Bianchi cosmological models. A series of papers [29, 45, 46, 47, $48,49,50,51,52,53,54,55,56]$ have studied on dynamical system analysis for the background of FRW metric. Recently several authors [46, 51, 57, 58, 59, $60,61,62,63,64]$ have studied phase-plane analysis of cosmological models in modified gravity. The dynamical systems analysis of anisotropic cosmological models with scalar fields are studied by $[65,66]$. In this paper, we use a different parametrisation of the equations of motion, which makes the connection between the curvature and dynamics more explicit.

The paper has the following structure. In Section 2 we will explore the model and basic equations for LRS BI cosmological universe. In section 3, we will discuss the dynamical evolution of linear interaction between dark sector of the universe by considering $Q=\sqrt{\frac{2}{3}} \kappa \beta \rho_{\gamma} \dot{\phi}$. The stability and viability of this model 
for linear coupling between both dark components are presented and discussed. In section 4 and 5, we shall explore the dynamics of interaction between dark sector by considering $Q=\alpha H \rho_{\gamma}$ and $Q=\Gamma \rho_{\gamma}$ respectively. We will also work out the phase plane analysis, local and classical stability analysis of critical points in respective sections for each models. Finally conclusions will be given in last Section 6.

\section{Model and Basic Equations}

For Locally Rotationally Symmetric (LRS) Bianchi type-I cosmological model, the metric is given by

$$
d s^{2}=d t^{2}-a_{1}^{2} d x^{2}-a_{2}^{2}\left(d y^{2}+d z^{2}\right)
$$

Here the metric $a_{1}(t), a_{2}(t)$ are expansion in the respective directions. The directional Hubble parameters are defined as

$$
H_{i}=\frac{\dot{a}_{i}}{a_{i}}, \quad i=1,2 .
$$

Here, dot denotes differential with respect to cosmic time $t$. By using Eq.(2), the Hubble parameter $H$ is given by

$$
H=\frac{\dot{a}}{a}=\frac{1}{3}\left(\frac{\dot{a_{1}}}{a_{1}}+2 \frac{\dot{a_{2}}}{a_{2}}\right)=\frac{1}{3}\left(H_{1}+2 H_{2}\right)
$$

Here we take an ansatz $a_{1} \propto a_{2}{ }^{n}, n>0$, LRS Bianchi type I model reduces to flat FRW cosmological model when $n=1$ whereas the model becomes anisotropic when $n \neq 1$.

From above assumption and Eq.(3), we can find a relation between $H_{i}(i=$ 1,2) and $H$ as

$$
H_{1}=n H_{2}=\left(\frac{3 n}{n+2}\right) H .
$$

The Einstein's field equations are written as

$$
G_{i j}=R_{i j}-\frac{1}{2} R g_{i j}=-\frac{8 \pi G}{c^{4}} T_{i j}
$$

Here, $R_{i j}, R$ and $g_{i j}$ are the Ricci tensor, Ricci scalar and metric tensor respectively. $T_{i j}$ is the energy momentum tensor consisting of dark sector and scalar field.

From Eqs.(1) and (5) the Einstein's field equations (5) are written as 


$$
\begin{aligned}
& 2 \frac{\dot{a_{1}} \dot{a_{2}}}{a_{1} a_{2}}-\left(\frac{\dot{a_{2}}}{a_{2}}\right)^{2}=\left(\rho_{\gamma}+\rho_{\phi}\right) \\
& 2 \frac{\ddot{a_{2}}}{a_{2}}+\left(\frac{\dot{a_{2}}}{a_{2}}\right)^{2}=-\left(p_{\gamma}+p_{\phi}\right) \\
& \frac{\ddot{a_{1}}}{a_{1}}+\frac{\ddot{a_{2}}}{a_{2}}+\frac{\dot{a_{1}} \dot{a_{2}}}{a_{1} a_{2}}=-\left(p_{\gamma}+p_{\phi}\right)
\end{aligned}
$$

Here $8 \pi G=c=1$ and we do not consider the baryons (which are not coupled to dark energy) and radiation (since we are mainly interested in the late universe). Here $\rho_{\gamma}$ and $p_{\gamma}$ are the energy density and pressure of dark sector of the universe respectively. The energy density and pressure of the scalar field are connected by an equation of state $p_{\phi}=\gamma_{\phi} \rho_{\phi}$, defined as

$$
\begin{aligned}
& \rho_{\phi}=\frac{1}{2} \dot{\phi}^{2}+V(\phi) \\
& p_{\phi}=\frac{1}{2} \dot{\phi}^{2}-V(\phi)
\end{aligned}
$$

By varying the action of the quintessence scalar field with respect to $\phi$ and dark sector coupling $Q$ is defined as

$$
\ddot{\phi}+\left(H_{1}+2 H_{2}\right) \dot{\phi}+\frac{d V}{d \phi}=\frac{Q}{\dot{\phi}}
$$

Here $Q$ is the rate of energy exchange in the dark sector. When $Q>0$ the energy transfer from dark matter to dark energy whereas the energy transfer is in reverse direction when $Q<0$.

A general coupling between a quintessence field $\phi$ and dark matter (with density $\rho_{\gamma}$ ) may be described in the background by the balance equations

$$
\begin{gathered}
\dot{\rho_{\gamma}}=-\left(H_{1}+2 H_{2}\right) \rho_{\gamma}-Q \\
\dot{\rho_{\phi}}=-\left(H_{1}+2 H_{2}\right)\left(1+\gamma_{\phi}\right) \rho_{\phi}+Q
\end{gathered}
$$

From Eqs.(4)-(10), the Friedmann and Raychaudhuri equations are written as

$$
\begin{gathered}
H^{2}=\frac{(n+2)^{2}}{9(2 n+1)}\left(\rho_{\gamma}+\frac{1}{2} \dot{\phi}^{2}+V(\phi)\right) \\
\dot{H}=-\frac{(n+2)^{2}}{6(2 n+1)}\left(\rho_{\gamma}+\dot{\phi}^{2}\right)
\end{gathered}
$$


Here, we the equation of state for dark energy is defined as $p_{\gamma}=\gamma \rho_{\gamma}$, where $\gamma(0 \leq \gamma \leq 1)$ is a constant.

We introduce two new dimensionless variables $x$ and $y$ as

$$
x^{2}=\frac{(n+2)^{2}}{18(2 n+1)}\left\{\frac{\dot{\phi}^{2}}{H^{2}}\right\} ; y^{2}=\frac{(n+2)^{2}}{9(2 n+1)}\left\{\frac{V(\phi)}{H^{2}}\right\}
$$

There is no as yet basis in fundamental theory for a specific coupling in the dark sector and therefore any coupling model will necessarily be phenomenological, although some models will have a more physical justification than others. Various models of energy exchange have been considered by [?]. A satisfactory model requires at least that $Q$ should be expressed in terms of the energy density and other covariant quantities. Here we will discuss three physically viable coupling model. Here we will discuss three physically viable coupling model.

\section{Coupling Model (I): $Q=\sqrt{\frac{2}{3}} \kappa \beta \rho_{\gamma} \dot{\phi}$}

Here $\beta$ is dimensionless constant whose sign determines the direction of energy transfer. When $\beta>0$, the energy transfer from dark matter to dark energy whereas when $\beta<0$, the energy transfer from dark energy to dark matter. Here we are considering exponential potential $V(\phi)=V_{0} e^{-\lambda \phi}$, where $\phi$ is the quintessence scalar field. Now we differentiate the above Eq.(16) with respect to the number of e-folding $N=\frac{1}{3} \ln \left(a_{1} a_{2}^{2}\right)$ then the system of autonomous equations are written as

$$
\begin{gathered}
\frac{d x}{d N} \equiv x^{\prime}=-3 x+\frac{3 \sqrt{(2 n+1)}}{\sqrt{2}(n+2)} \lambda y^{2}+\frac{3}{2} x\left(1+x^{2}-y^{2}\right)+\frac{\sqrt{3} \sqrt{(2 n+1)}}{n+2} \beta\left(1+x^{2}-y^{2}\right) \\
\frac{d y}{d N} \equiv y^{\prime}=-\lambda \frac{3 \sqrt{(2 n+1)}}{\sqrt{2}(n+2)} x y+\frac{3}{2} y\left(1+x^{2}-y^{2}\right)
\end{gathered}
$$

From Eqs. (14) and (16), one can obtain

$$
1-x^{2}-y^{2}=\frac{(n+2)^{2}}{9(2 n+1)}\left\{\frac{\rho_{\gamma}}{H^{2}}\right\}
$$

Using constraint equation in above Eq.(19), we get

$$
\Omega_{\phi} \equiv \frac{\rho_{\gamma}(n+2)^{2}}{9 H^{2}(2 n+1)}=x^{2}+y^{2}
$$


Here $0 \leq \Omega_{\phi} \leq 1$. For scalar field, the effective equation of state is defined as

$$
\gamma_{\phi}=\frac{p_{\phi}}{\rho_{\phi}}
$$

Using Eqs. (9), (10), (16) in Eq.(21), we get

$$
\gamma_{\phi}=\frac{x^{2}-y^{2}}{x^{2}+y^{2}}
$$

The deceleration parameter ' $q$ ' is given as

$$
q=\frac{d}{d t}\left(\frac{1}{H}\right)-1=-1-\frac{\dot{H}}{H^{2}}=-1+\frac{3}{2}\left(1+x^{2}-y^{2}\right)
$$

\subsection{Phase-space analysis of model I}

To study the dynamical system (Eqs. $17 \& 18$ ), we firstly find the fixed/ critical points of the system. The critical points of the system are the simultaneous solutions of the equations

$$
\frac{d x}{d N}=0, \quad \frac{d y}{d N}=0 .
$$

To study the phase space behaviour of the system, we analyse the stability of fixed points by finding eigenvalues of the matrix, evaluated at the fixed point $\left(x_{\star}, y_{\star}\right)$ :

$$
M=\left[\begin{array}{ll}
\frac{\partial f(x, y)}{\partial x} & \frac{\partial f(x, y)}{\partial y} \\
\frac{\partial g(x, y)}{\partial x} & \frac{\partial g(x, y)}{\partial y}
\end{array}\right]
$$

where $f(x, y)=x^{\prime}$ and $g(x, y)=y^{\prime}$

From Eqs. (17\&18), the autonomous system of equations have five critical points namely $\mathrm{A}, \mathrm{B}, \mathrm{C}, \mathrm{D}$ and $\mathrm{E}$ :

- Point A $(1,0)$

- Point B $(-1,0)$

- Point C $\left(\frac{2 \beta k^{\prime}}{3}, 0\right)$

- Point D $\left(\frac{l^{\prime} \lambda}{3},\left(1-\left(\frac{l^{\prime} \lambda}{3}\right)^{2}\right)^{\frac{1}{2}}\right)$

- Point E $\left(-\frac{3}{2\left(\beta k^{\prime}-\lambda l^{\prime}\right)}, \frac{\left(4 \beta^{2} k^{\prime 2}-4 \beta \lambda l^{\prime} k^{\prime}+9\right)^{\frac{1}{2}}}{2\left(\beta k^{\prime}-\lambda l^{\prime}\right)}\right)$ 


\begin{tabular}{|c|c|c|c|c|c|c|}
\hline Point & $x_{\star}$ & $y_{\star}$ & $\Omega_{\phi}$ & $\gamma_{\phi}$ & $q$ & Existenc \\
\hline A & 1 & 0 & 1 & 1 & 2 & $\forall \lambda, \gamma$ \\
\hline $\mathrm{B}$ & -1 & 0 & 1 & 1 & 2 & $\overline{\forall \lambda, \gamma}$ \\
\hline $\mathrm{C}$ & $\frac{2 \beta k^{\prime}}{3}$ & 0 & $\frac{4 \beta^{2} k^{\prime 2}}{9}$ & 1 & $\frac{1}{2}+\frac{2}{3} \beta^{2} k^{\prime 2}$ & $\begin{array}{c}\beta \leq \\
\frac{\sqrt{3}(n+2)}{2 \sqrt{2 n+1}}\end{array}$ \\
\hline $\mathrm{D}$ & $\frac{l^{\prime} \lambda}{3}$ & $\left(1-\frac{l^{\prime 2} \lambda^{2}}{9}\right)^{1 / 2}$ & 1 & $\frac{2 l^{\prime 2} \lambda^{2}}{9}-1$ & $-1+\frac{l^{\prime 2} \lambda^{2}}{3}$ & $\begin{array}{c}\lambda \leq \\
\frac{\sqrt{2}(n+2)}{\sqrt{2 n+1}}\end{array}$ \\
\hline $\mathrm{E}$ & $\frac{3}{2\left(\lambda l^{\prime}-\beta k^{\prime}\right)}$ & $\frac{\sqrt{4 \beta^{2} k^{\prime 2}-4 \beta \lambda l^{\prime} k^{\prime}+9}}{2\left(\beta k^{\prime}-\lambda l^{\prime}\right)}$ & $\frac{2 \beta^{2} k^{\prime 2}-2 \beta \lambda l^{\prime} k^{\prime}+9}{2\left(\beta k^{\prime}-\lambda l^{\prime}\right)^{2}}$ & $\frac{2 \beta k^{\prime}\left(\lambda l^{\prime}-\beta k^{\prime}\right)}{2 \beta^{2} k^{\prime 2}-2 \beta \lambda l^{\prime} k^{\prime}+9}$ & $\frac{l^{\prime 2} \lambda^{2}-2 \beta^{2} k^{\prime 2}+l^{\prime} k^{\prime} \lambda \beta}{\left(\beta k^{\prime}-\lambda l^{\prime}\right)^{2}}$ & $\begin{array}{l}\lambda \geq \\
\frac{\sqrt{3}(n+2)}{\sqrt{2 n+1}} \\
+\frac{2}{\sqrt{3} \beta}\end{array}$ \\
\hline
\end{tabular}

Table 1: Physical parameters at fixed points for model I.

\begin{tabular}{|c|c|c|c|c|}
\hline Point & $x_{\star}$ & $y_{\star}$ & $\lambda_{1}$ & $\lambda_{2}$ \\
\hline \hline $\mathrm{A}$ & 1 & 0 & $3-2 \beta k^{\prime}$ & $3-\lambda l^{\prime}$ \\
\hline $\mathrm{B}$ & -1 & 0 & $3+2 \beta k^{\prime}$ & $3+\lambda l^{\prime}$ \\
\hline $\mathrm{C}$ & $\frac{2 \beta k^{\prime}}{3}$ & 0 & $-\frac{3}{2}+\frac{2 \beta^{2} k^{\prime 2}}{3}$ & $\frac{3}{2}-\frac{2 \beta k^{\prime}}{3}\left(\lambda l^{\prime}-\beta k^{\prime}\right)$ \\
\hline $\mathrm{D}$ & $\frac{l^{\prime} \lambda}{3}$ & $\left(1-\frac{l^{\prime 2} \lambda^{2}}{9}\right)^{1 / 2}$ & $-3+\frac{\lambda^{2} l^{\prime 2}}{3}$ & $-3-\frac{2 \lambda l^{\prime}}{3}\left(\beta k^{\prime}-\lambda l^{\prime}\right)$ \\
\hline $\mathrm{E}$ & $-\frac{3}{2\left(\beta k^{\prime}-\lambda l^{\prime}\right)}$ & $\frac{\left(4 \beta^{2} k^{\prime 2}-4 \beta \lambda l^{\prime} k^{\prime}+9\right)^{1 / 2}}{2\left(\beta k^{\prime}-\lambda l^{\prime}\right)}$ & $-\frac{\left(3 \lambda l^{\prime}-2\left(X_{E}\right)^{1 / 2}\right)}{4\left(\beta k^{\prime}-\lambda l^{\prime}\right)}-\frac{3}{2}$ & $-\frac{\left(3 \lambda l^{\prime}+2\left(X_{E}\right)^{1 / 2}\right)}{4\left(\beta k^{\prime}-\lambda l^{\prime}\right)}-\frac{3}{2}$ \\
\hline
\end{tabular}

Table 2: Critical points and eigenvalues for model I.

where $k^{\prime}=\frac{\sqrt{3} \sqrt{(2 n+1)}}{n+2}$ and $l^{\prime}=\frac{3 \sqrt{2 n+1}}{\sqrt{2}(n+2)}$ i.e. $k^{\prime}=\frac{\sqrt{2}}{\sqrt{3}} l^{\prime}$. The cosmological parameters at critical points are given in Table 1 .

where $X_{E}=8 \beta^{3} \lambda k^{\prime 3} l^{\prime}-16 \beta^{2} \lambda^{2} k^{\prime 2} l^{\prime 2}+45 \beta^{2} k^{\prime 2}+8 \beta \lambda^{3} k^{\prime} l^{\prime 3}-27 \beta \lambda k^{\prime} l^{\prime}-$ $\frac{63 \lambda^{2} l^{\prime 2}}{4}+81$.

Here it is observed that, from Table 1, the critical points A and B are always exist for all values of $\lambda$ and $\gamma$. The critical points $C, D$ and $E$ exist when the restrictions are $\beta \leq \frac{\sqrt{3}(n+2)}{2 \sqrt{2 n+1}}, \lambda \leq \frac{\sqrt{2}(n+2)}{\sqrt{2 n+1}}$ and $\lambda \geq \frac{\sqrt{3}(n+2)}{\sqrt{2 n+1}}+\frac{2}{\sqrt{3} \beta}$ respectively. The nature of critical points and eigenvalues of the Jacobian matrix at critical points are presented in Table 2.

\subsection{Local and classical stability analysis of model I}

In this section, we will discuss the local and classical stability of model I

Nature of point A, unstable point if $\beta<\frac{\sqrt{3}(n+2)}{2 \sqrt{2 n+1}}$ and $\lambda<\frac{\sqrt{2 n+1}}{\sqrt{2}(n+2)}$ whereas 
this point becomes stable if $\beta>\frac{\sqrt{3}(n+2)}{2 \sqrt{2 n+1}}$ and $\lambda>\frac{\sqrt{2 n+1}}{\sqrt{2}(n+2)}$. This critical point is saddle (unstable) if either $\beta>\frac{\sqrt{3}(n+2)}{2 \sqrt{2 n+1}}$ and $\lambda<\frac{\sqrt{2 n+1}}{\sqrt{2}(n+2)}$ or $\beta<\frac{\sqrt{3}(n+2)}{2 \sqrt{2 n+1}}$ and $\lambda>\frac{\sqrt{2 n+1}}{\sqrt{2}(n+2)}$. From Eq.(20), we can obtain $\Omega_{\phi}=1$ at point A, this means the kinetic dominated solution exists near this point. From Eq.(23), one can obtain $q=2$ (i.e. decelerating phase of the universe) and the average scale factor of the model is evolves as $a \propto t^{\frac{1}{3}}$ at this point A. Using above relation of scale factor with Eqs.(2) and (3), the directional Hubble parameters $H_{1}$ and $H_{2}$ are given by $\frac{n}{(n+2) t}$ and $\frac{1}{(n+2) t}$ respectively. In view of Eq.(22) with $x=1 \& y=0$, the effective equation of state parameter $\gamma_{\phi}$ is equal to one.

Nature of point $\mathrm{B}$, unstable point if $\beta>-\frac{\sqrt{3}(n+2)}{2 \sqrt{2 n+1}}$ and $\lambda>-\frac{\sqrt{2}(n+2)}{\sqrt{2 n+1}}$ whereas this point becomes stable if $\beta<-\frac{\sqrt{3}(n+2)}{2 \sqrt{2 n+1}}$ and $\lambda<-\frac{\sqrt{2}(n+2)}{\sqrt{2 n+1}}$. This critical point is saddle (unstable) if either $\beta<-\frac{\sqrt{3}(n+2)}{2 \sqrt{2 n+1}}$ and $\lambda>-\frac{\sqrt{2}(n+2)}{\sqrt{2 n+1}}$ or $\beta>-\frac{\sqrt{3}(n+2)}{2 \sqrt{2 n+1}}$ and $\lambda<-\frac{\sqrt{2}(n+2)}{\sqrt{2 n+1}}$. From Eq.(20), we can obtain $\Omega_{\phi}=1$ at point B, this means the kinetic dominated solution exists near this point. From Eq.(23), one can obtain $q=2$ (i.e. decelerating phase of the universe) and the average scale factor of the model is evolves as $a \propto t^{\frac{1}{3}}$ at this point B. Using above relation of scale factor with Eqs.(2) and (3), the directional Hubble parameters $H_{1}$ and $H_{2}$ are given by $\frac{n}{(n+2) t}$ and $\frac{1}{(n+2) t}$ respectively. In view of Eq. (22) with $x=-1 \& y=0$, the effective equation of state parameter $\gamma_{\phi}$ is equal to one.

Nature of point $\mathbf{C}$, unstable point if $\beta^{2}>\frac{3(n+2)^{2}}{4(2 n+1)}$ and $\lambda<\frac{\sqrt{3}(n+2)^{2}}{2 \sqrt{2}(2 n+1) \beta}+\frac{\sqrt{2}}{\sqrt{3}} \beta$ whereas this point becomes stable if $\beta^{2}<\frac{3(n+2)^{2}}{4(2 n+1)}$ and $\lambda>\frac{\sqrt{3}(n+2)^{2}}{2 \sqrt{2}(2 n+1) \beta}+\frac{\sqrt{2}}{\sqrt{3}} \beta$. This critical point is Saddle (unstable) point if either $\beta^{2}>\frac{3(n+2)^{2}}{4(2 n+1)}$ and $\lambda>$ $\frac{\sqrt{3}(n+2)^{2}}{2 \sqrt{2}(2 n+1) \beta}+\frac{\sqrt{2}}{\sqrt{3}} \beta$ or $\beta^{2}<\frac{3(n+2)^{2}}{4(2 n+1)}$ and $\lambda<\frac{\sqrt{3}(n+2)^{2}}{2 \sqrt{2}(2 n+1) \beta}+\frac{\sqrt{2}}{\sqrt{3}} \beta$. From Eq.(23), one can obtain there is no accelerating phase of the universe near point $\mathrm{C}$ for all values of $\beta$ and $n$ and the average scale factor of the model is evolves as $a \propto \frac{3}{2}\left(1+\frac{4 \beta^{2} k^{\prime 2}}{9}\right) t^{\frac{2}{3\left(1+\frac{4 \beta^{2} k^{\prime 2}}{9}\right)}}$ at this point C. Using above relation of scale factor with Eqs.(2) and (3), the directional Hubble parameters $H_{1}$ and $H_{2}$ are given by $\frac{2 n}{(n+2)\left(1+\frac{4 \beta^{2} k^{\prime 2}}{9}\right) t}$ and $\frac{2}{(n+2)\left(1+\frac{4 \beta^{2} k^{\prime 2}}{9}\right) t}$ respectively. In view of Eq.(22), the effective equation of state parameter $\gamma_{\phi}$ is equal to one at point $\mathrm{C}$.

Nature of the point $\mathrm{D}$, unstable point if $\lambda^{2}>\frac{2(n+2)^{2}}{(2 n+1)}$ and $\beta<-\frac{\sqrt{3}}{\sqrt{2 \lambda}}\left(\frac{n^{2}+3}{2 n+1}\right)$ whereas this point becomes stable if $\lambda^{2}<\frac{2(n+2)^{2}}{(2 n+1)}$ and $\beta>-\frac{\sqrt{3}}{\sqrt{2} \lambda}\left(\frac{n^{2}+3}{2 n+1}\right)$. This critical point is saddle (unstable) if either $\lambda^{2}<\frac{2(n+2)^{2}}{(2 n+1)}$ and $\beta<-\frac{\sqrt{3}}{\sqrt{2 \lambda}}\left(\frac{n^{2}+3}{2 n+1}\right)$ or $\lambda^{2}>\frac{2(n+2)^{2}}{(2 n+1)}$ and $\beta>-\frac{\sqrt{3}}{\sqrt{2} \lambda}\left(\frac{n^{2}+3}{2 n+1}\right)$. From Eq.(23), one can obtain there is no accelerating phase of the universe near point $\mathrm{D}$ for $\lambda^{2} \geq \frac{2(n+2)^{2}}{(2 n+1)}$ while go to 
accelerating phase when $\lambda^{2}<\frac{2(n+2)^{2}}{(2 n+1)}$ and the average scale factor of the model is evolves as $a \propto \frac{\lambda^{2} l^{2}}{3} t \frac{3}{\lambda^{2} l^{\prime 2}}$ at this point. Using above relation of scale factor with Eqs.(2) and (3), the directional Hubble parameters $H_{1}$ and $H_{2}$ are given by $\frac{9 n}{(n+2) \lambda^{2} l^{\prime 2} t}$ and $\frac{9}{(n+2) \lambda^{2} l^{\prime 2} t}$ respectively. In view of Eq.(22), the effective equation of state parameter is given by $\gamma_{\phi}=\frac{(2 n+1)}{(n+2)} \lambda^{2}-1$ at point $\mathrm{D}$.

Nature of the point $\mathrm{E}$, unstable point if $\left(X_{E}\right)^{1 / 2}>-\frac{3}{2}\left(\lambda l^{\prime}-2 \beta k^{\prime}\right)$ and $\left(X_{E}\right)^{1 / 2}<$ $\frac{3}{2}\left(\lambda l^{\prime}-2 \beta k^{\prime}\right)$ where as this point becomes stable if $\left(X_{E}\right)^{1 / 2}<-\frac{3}{2}\left(\lambda l^{\prime}-2 \beta k^{\prime}\right)$ and $\left(X_{E}\right)^{1 / 2}>\frac{3}{2}\left(\lambda l^{\prime}-2 \beta k^{\prime}\right)$. This critical point is Saddle (unstable) if either $\left(X_{E}\right)^{1 / 2}>-\frac{3}{2}\left(\lambda l^{\prime}-2 \beta k^{\prime}\right)$ and $\left(X_{E}\right)^{1 / 2}>\frac{3}{2}\left(\lambda l^{\prime}-2 \beta k^{\prime}\right)$ or $\left(X_{E}\right)^{1 / 2}<$ $-\frac{3}{2}\left(\lambda l^{\prime}-2 \beta k^{\prime}\right)$ and $\left(X_{E}\right)^{1 / 2}<\frac{3}{2}\left(\lambda l^{\prime}-2 \beta k^{\prime}\right)$. From Eq. (23), one can obtain there is accelerating phase of the universe near point $\mathrm{E}$ for $\lambda<\frac{8 \beta}{\sqrt{3}(4+\sqrt{2})}$ and the average scale factor of the model is evolves as $a \propto \frac{3}{2} \frac{\left(\lambda^{2} l^{\prime 2}-\beta \lambda l^{\prime} k^{\prime}\right)}{\left(\beta k^{\prime}-\lambda l^{\prime}\right)^{2}} t^{\frac{2}{3}} \frac{\left(\beta k^{\prime}-\lambda l^{\prime}\right)^{2}}{\left(\lambda^{2} l^{\prime 2}-\beta \lambda l^{\prime} k^{\prime}\right)}$ at this point. Using above relation of scale factor with Eqs.(2) and (3), the directional Hubble parameters $H_{1}$ and $H_{2}$ are given by $\frac{2 n\left(\beta k^{\prime}-\lambda l^{\prime}\right)^{2}}{(n+2)\left(\lambda^{2} l^{\prime 2}-\beta \lambda l^{\prime} k^{\prime}\right) t}$ and $\frac{2\left(\beta k^{\prime}-\lambda l^{\prime}\right)^{2}}{(n+2)\left(\lambda^{2} l^{\prime 2}-\beta \lambda l^{\prime} k^{\prime}\right) t}$ respectively. In view of Eq.(22), the effective equation of state parameter is given by $\gamma_{\phi}=-\frac{2 \beta k^{\prime}\left(\beta k^{\prime}-\lambda l^{\prime}\right)}{2 \beta^{2} k^{\prime 2}-2 \beta \lambda l^{\prime} k^{\prime}+9}$ at point $\mathrm{E}$.

In view of classical stability, the model is stable when speed of sound $\left(C_{s}^{2}\right)$ holds the condition $0 \leq C_{s}^{2} \leq 1$. In the present cosmological scenario we have

$$
C_{s}^{2}=\frac{2 x^{2}-\frac{2}{\sqrt{3}} \frac{\sqrt{2 n+1}}{n+2} \beta x\left(1-x^{2}-y^{2}\right)-\frac{2 \sqrt{2} \sqrt{2 n+1}}{n+2} \lambda x y^{2}}{1+x^{2}-y^{2}}
$$

We have given both local and classical stability for model I in Table 3. It is observed that points $\mathrm{A}$ and $\mathrm{B}$ are not locally stable when $\beta<\frac{\sqrt{3}(n+2)}{2 \sqrt{2 n+1}} ; \lambda<$ $\frac{\sqrt{2 n+1}}{\sqrt{2}(n+2)}$ and $\beta>-\frac{\sqrt{3}(n+2)}{2 \sqrt{2 n+1}} ; \lambda>-\frac{\sqrt{2}(n+2)}{\sqrt{2 n+1}}$ respectively while these points are classically stable for all $\lambda$ and $\beta$. It is also observed that point $\mathrm{C}$ is locally unstable when $\beta^{2}>\frac{3(n+2)^{2}}{4(2 n+1)}$ and $\lambda<\frac{\sqrt{3}(n+2)^{2}}{2 \sqrt{2}(2 n+1) \beta}+\frac{\sqrt{2}}{\sqrt{3}} \beta$ but classically stable when $\beta^{2}<$ $\frac{3(n+2)^{2}}{4(2 n+1)}$. From Table 3, it is noted that point D is stable when $\lambda^{2}<\frac{2(n+2)^{2}}{(2 n+1)}$ and $\beta>-\frac{\sqrt{3}}{\sqrt{2} \lambda}\left(\frac{n^{2}+3}{2 n+1}\right)$ while this point corresponds to classical stability when $\lambda<$ $\frac{\sqrt{2 n+1}}{\sqrt{2}(n+2)}$. It is interesting that the point $\mathrm{E}$ is locally and classically stable under some restrictions on $\lambda$ and $\beta$. 


\begin{tabular}{|c|c|c|c|c|}
\hline Point & $x_{\star}$ & Drat & Local stability & Classical stability \\
\hline $\mathrm{A}$ & $\bar{~} 1$ & 3 & $\begin{array}{c}\text { Stable point if } \beta>\frac{\sqrt{3}(n+2)}{2 \sqrt{2 n+1}} \\
\text { and } \lambda>\frac{\sqrt{2 n+1}}{\sqrt{2}(n+2)} \\
\text { Unstable otherwise }\end{array}$ & Stable \\
\hline B & -1 & 0 & $\begin{array}{c}\text { Stable point if } \beta<-\frac{\sqrt{3}(n+2)}{2 \sqrt{2 n+1}} \\
\text { and } \lambda<-\frac{\sqrt{2}(n+2)}{\sqrt{2 n+1}} \\
\text { Unstable otherwise }\end{array}$ & Stable \\
\hline $\mathrm{C}$ & $\frac{2 \beta k^{\prime}}{3}$ & 0 & $\begin{array}{c}\text { Stable point if } \beta^{2}<\frac{3(n+2)^{2}}{4(2 n+1)} \\
\text { and } \lambda>\frac{\sqrt{3}(n+2)^{2}}{2 \sqrt{2}(2 n+1) \beta}+\frac{\sqrt{2}}{\sqrt{3}} \beta \\
\text { Unstable otherwise }\end{array}$ & $\begin{array}{c}\text { Stable } \\
\text { if } \beta^{2}<\frac{3(n+2)^{2}}{4(2 n+1)}\end{array}$ \\
\hline $\mathrm{D}$ & $\frac{l^{\prime} \lambda}{3}$ & $\left(1-\frac{l^{\prime 2} \lambda^{2}}{9}\right)^{1 / 2}$ & $\begin{array}{c}\text { Stable point if } \lambda^{2}<\frac{2(n+2)^{2}}{(2 n+1)} \\
\text { and } \beta>-\frac{\sqrt{3}}{\sqrt{2} \lambda}\left(\frac{n^{2}+3}{2 n+1}\right)\end{array}$ & $\begin{array}{c}\text { Stable if } \\
\lambda<\frac{\sqrt{2}(n+2)}{\sqrt{2 n+1}}\end{array}$ \\
\hline $\mathrm{E}$ & $-\frac{3}{2\left(\beta k^{\prime}-\lambda l^{\prime}\right)}$ & $\frac{\left(4 \beta^{2} k^{\prime 2}-4 \beta \lambda l^{\prime} k^{\prime}+9\right)^{1 / 2}}{2\left(\beta k^{\prime}-\lambda l^{\prime}\right)}$ & $\begin{array}{c}\text { Stable point if } \\
\left(X_{E}\right)^{1 / 2}<-\frac{3}{2}\left(\lambda l^{\prime}-2 \beta k^{\prime}\right) \\
\text { and }\left(X_{E}\right)^{1 / 2}>\frac{3}{2}\left(\lambda l^{\prime}-2 \beta k^{\prime}\right)\end{array}$ & $\begin{array}{c}\text { Stable if } \\
-4 \beta^{2}+3 \sqrt{6} \lambda \beta \\
-3 \lambda^{2} \leq 0\end{array}$ \\
\hline
\end{tabular}

Table 3: Local and classical stability criteria at fixed points for the model-I. 


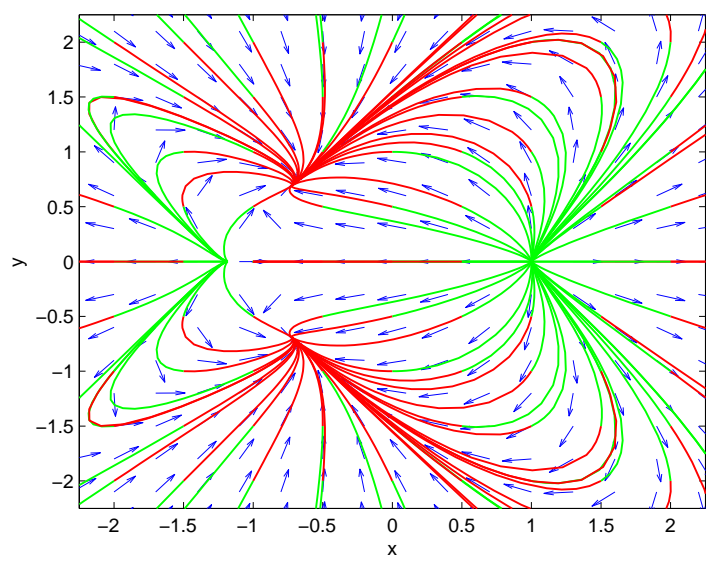

Figure 1: Phase plot of the system (model-I) when $n=0.05, \lambda=-2, \beta=-2$,

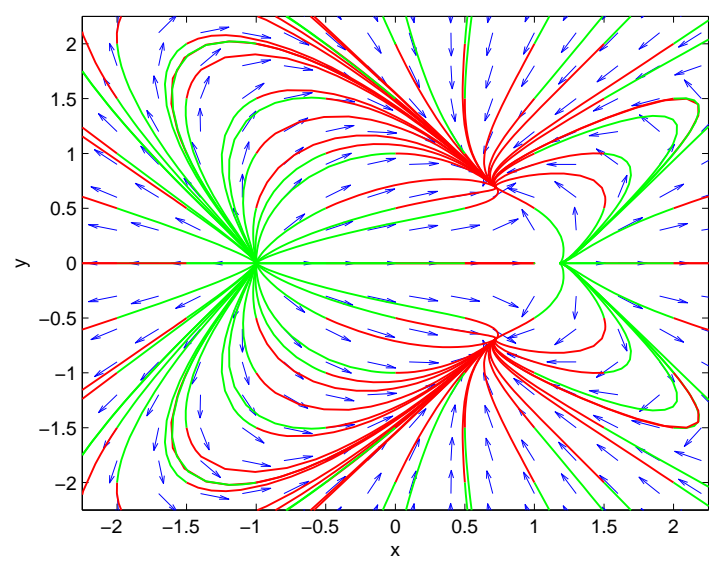

Figure 2: Phase plot of the system (model-I) when $n=0.05, \lambda=2, \beta=2$,

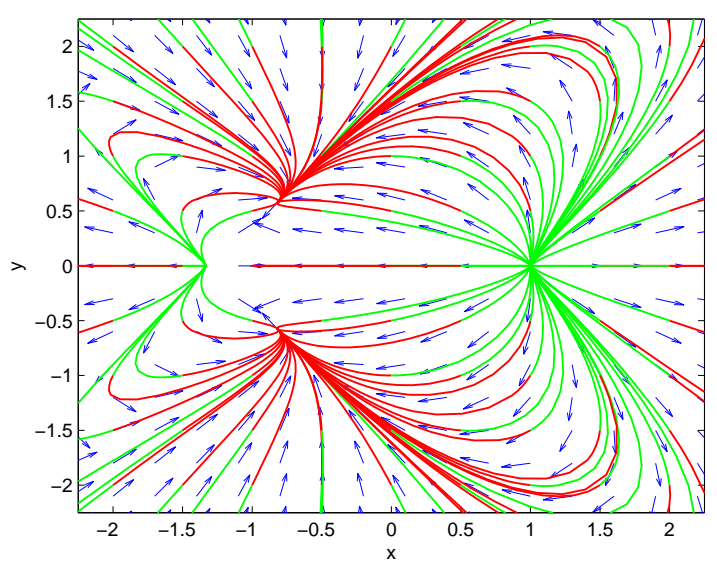

Figure 3: Phase plot of the system (model-I) when $n=1, \lambda=-2, \beta=-2$, 11 


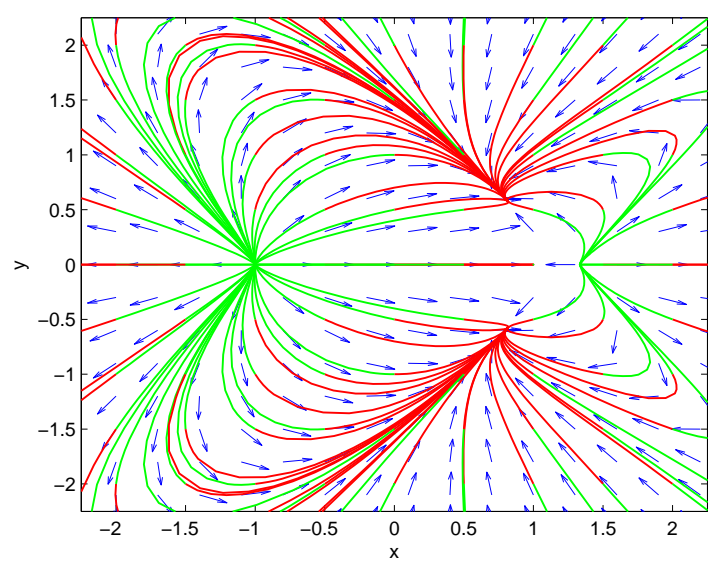

Figure 4: Phase plot of the system (model-I) when $n=1, \lambda=2, \beta=2$,

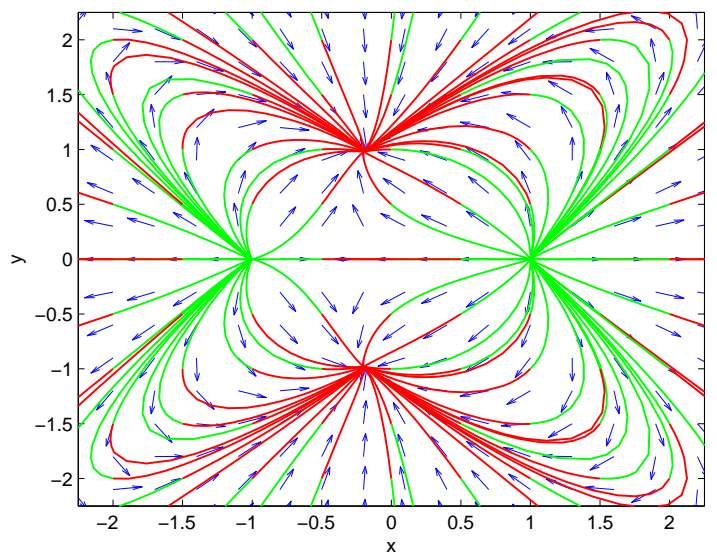

Figure 5: Phase plot of the system (model-I) when $n=100, \lambda=-2, \beta=-2$,

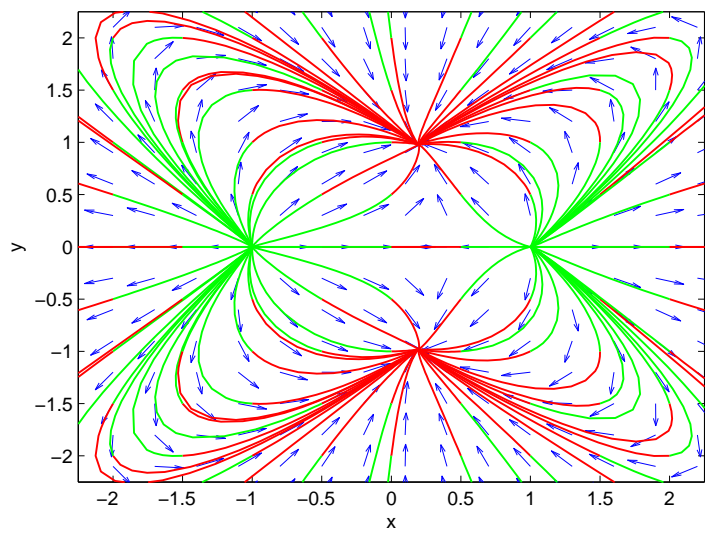

Figure 6: Phase plot of the system (model-I) when $n=100, \lambda=2, \beta=2$, 


\section{Coupling Model (II): $Q=\alpha H \rho_{\gamma}$}

Here $\alpha$ is dimensionless constant whose sign determines the direction of energy transfer. When $\alpha>0$, the energy transfer from dark matter to dark energy whereas when $\alpha<0$, the energy transfer from dark energy to dark matter.

In this Section, we are considering the dark sector interaction as $Q=\alpha H \rho_{\gamma}$ for LRS BI cosmological model, while still keeping the same variables as mentioned in Sec. 3.

The autonomous system of equations for model II are

$$
\begin{gathered}
\frac{d x}{d N} \equiv x^{\prime}=-3 x+\frac{3 \sqrt{(2 n+1)}}{\sqrt{2}(n+2)} \lambda y^{2}+\frac{3}{2} x\left(1+x^{2}-y^{2}\right)+\frac{\alpha\left(1-x^{2}-y^{2}\right)}{2 x} \\
\frac{d y}{d N} \equiv y^{\prime}=-\lambda \frac{3 \sqrt{(2 n+1)}}{\sqrt{2}(n+2)} x y+\frac{3}{2} y\left(1+x^{2}-y^{2}\right)
\end{gathered}
$$

\subsection{Phase-space analysis of model II}

From Eqs. (27\&28), the autonomous system of equations have six critical points namely A,B,C,D,E and F:

- Point A $(1,0)$

- Point B $(-1,0)$

- Point $\mathrm{C}\left(\frac{\sqrt{\alpha}}{\sqrt{3}}, 0\right)$

- Point D $\left(-\frac{\sqrt{\alpha}}{\sqrt{3}}, 0\right)$

- Point E $\left(\frac{l^{\prime} \lambda}{3},\left(1-\left(\frac{l^{\prime} \lambda}{3}\right)^{2}\right)^{1 / 2}\right)$

- Point $\mathrm{F}\left(\frac{\alpha+3}{2 \lambda l^{\prime}}, \frac{\left((\alpha+3)^{2}-\frac{4 \alpha \lambda^{2} l^{\prime 2}}{3}\right)^{1 / 2}}{2 \lambda l^{\prime}}\right)$

where $k^{\prime}=\frac{\sqrt{2}}{\sqrt{3}} l^{\prime}=\frac{\sqrt{3} \sqrt{(2 n+1)}}{n+2}$.

where $X_{F}=-3 \alpha^{5}-\frac{15 \lambda^{2} \alpha^{4} l^{\prime 2}}{4}+45 \alpha^{4}+\frac{2 \alpha^{3} \lambda^{4} l^{4}}{3}-51 \alpha^{3} \lambda^{2} l^{\prime 2}+270 \alpha^{3}+\frac{4 \alpha^{2} \lambda^{6} l^{\prime 6}}{9}+$ $12 \alpha^{2} \lambda^{4} l^{4}-\frac{441 \alpha^{2} \lambda^{2} l^{\prime 2}}{2}+810 \alpha^{2}+30 \alpha \lambda^{4} l^{\prime 4}-351 \alpha \lambda^{2} l^{\prime 2}+1215 \alpha-\frac{567 \lambda^{2} l^{\prime 2}}{4}+729$.

Here it is noted that from Table 4 , the critical points A and B are always exist for all values of $\lambda$ and $\gamma$, while the critical points $\mathrm{C}$ and $\mathrm{D}$ exist for $0 \leq \alpha \leq 3$. The other critical points E \& F exist when $\lambda \leq \frac{\sqrt{2}(n+2)}{\sqrt{2 n+1}}$ and $\lambda \geq \frac{(n+2)(\alpha+3)}{3 \sqrt{2} \sqrt{2 n+1}}$ respectively. The nature of critical points and eigenvalues of the jacobian matrix at critical points are given in Table 5. 


\begin{tabular}{|c|c|c|c|c|c|c|}
\hline Point & $x_{\star}$ & $y_{\star}$ & $\Omega_{\phi}$ & $\gamma_{\phi}$ & $q$ & Existence \\
\hline \hline A & 1 & 0 & 1 & 1 & 2 & $\forall \lambda, \gamma$ \\
\hline B & -1 & 0 & 1 & 1 & 2 & $\forall \lambda, \gamma$ \\
\hline C & $\frac{\sqrt{\alpha}}{\sqrt{3}}$ & 0 & $\frac{\alpha}{3}$ & 1 & $\frac{1+\alpha}{2}$ & $0 \leq \alpha \leq 3$ \\
\hline D & $-\frac{\sqrt{\alpha}}{\sqrt{3}}$ & 0 & $\frac{\alpha}{3}$ & 1 & $\frac{1+\alpha}{2}$ & $0 \leq \alpha \leq 3$ \\
\hline E & $\frac{\lambda l^{\prime}}{3}$ & $\left(1-\left(\frac{\lambda l^{\prime}}{3}\right)^{2}\right)^{1 / 2}$ & 1 & $-1+\frac{2 \lambda^{2} l^{\prime 2}}{9}$ & $-1+\frac{\lambda^{2} l^{\prime 2}}{3}$ & $\lambda \leq \frac{\sqrt{2}(n+2)}{\sqrt{2 n+1}}$ \\
\hline F & $\frac{\alpha+3}{2 \lambda l^{\prime}}$ & $\frac{\left((\alpha+3)^{2}-\frac{4 \alpha \lambda^{2} l^{\prime 2}}{3}\right)^{1 / 2}}{2 \lambda l^{\prime}}$ & $\frac{2(\alpha+3)^{2}-\frac{4 \alpha \lambda^{2} l^{\prime 2}}{3}}{4 \lambda^{2} l^{\prime 2}}$ & $\frac{4 \alpha \lambda^{2} l^{\prime 2}}{6(\alpha+3)^{2}-4 \alpha \lambda^{2} l^{\prime 2}}$ & $\frac{1+3 \alpha}{2}$ & $\lambda \geq \frac{(n+2)(\alpha+3)}{3 \sqrt{2} \sqrt{2 n+1}}$ \\
\hline
\end{tabular}

Table 4: Physical parameters at fixed points for model II.

\begin{tabular}{|c|c|c|c|c|}
\hline Point & $x_{\star}$ & $y_{\star}$ & $\lambda_{1}$ & $\lambda_{2}$ \\
\hline \hline $\mathrm{A}$ & 1 & 0 & $3-\alpha$ & $3-\lambda l^{\prime}$ \\
\hline $\mathrm{B}$ & -1 & 0 & $3-2 \alpha$ & $3+\lambda l^{\prime}$ \\
\hline $\mathrm{C}$ & $\frac{\sqrt{\alpha}}{\sqrt{3}}$ & 0 & $-3+\alpha$ & $\frac{\alpha+3}{2}-\lambda l^{\prime}\left(\frac{\alpha}{3}\right)^{1 / 2}$ \\
\hline $\mathrm{D}$ & $-\frac{\sqrt{\alpha}}{\sqrt{3}}$ & 0 & $-3+\alpha$ & $\frac{\alpha+3}{2}+\lambda l^{\prime}\left(\frac{\alpha}{3}\right)^{1 / 2}$ \\
\hline $\mathrm{E}$ & $\frac{\lambda l^{\prime}}{3}$ & $\left(1-\left(\frac{\lambda l^{\prime}}{3}\right)^{2}\right)^{1 / 2}$ & $-3+\frac{\lambda^{2} l^{\prime 2}}{3}$ & $-(\alpha+3)+\frac{2 \lambda^{2} l^{\prime 2}}{3}$ \\
\hline $\mathrm{F}$ & $\frac{\alpha+3}{2 \lambda l^{\prime}}$ & $\frac{\left((\alpha+3)^{2}-\frac{4 \alpha \lambda^{2} l^{\prime 2}}{3}\right)^{1 / 2}}{2 \lambda l^{\prime}}$ & $\frac{\left(X_{F}\right)^{1 / 2}}{6 \lambda l^{\prime}+2 \alpha \lambda l^{\prime}}+\frac{3 \alpha^{2}+6 \alpha-9}{12+4 \alpha}-\frac{\alpha \lambda^{2} l^{\prime 3}}{9+3 \alpha}$ & $-\frac{\left(X_{F}\right)^{1 / 2}}{6 \lambda l^{\prime}+2 \alpha \lambda l^{\prime}}+\frac{3 \alpha^{2}+6 \alpha-9}{12+4 \alpha}-\frac{\alpha \lambda^{2} l^{\prime 3}}{9+3 \alpha}$ \\
\hline
\end{tabular}

Table 5: Critical points and eigenvalues for model II. 


\subsection{Local and classical stability analysis of model II}

In this section we are discussing the stability of the critical points A,B,C, D, E and F of the autonomous system of Eqs. $(27 \& 28)$.

Nature of point A, unstable if $\alpha<3$ and $\lambda<\frac{\sqrt{2}(n+2)}{\sqrt{2 n+1}}$ whereas this point becomes stable if $\alpha>3$ and $\lambda>\frac{\sqrt{2}(n+2)}{\sqrt{2 n+1}}$. This critical point A is saddle if either $\alpha<3$ and $\lambda>\frac{\sqrt{2}(n+2)}{\sqrt{2 n+1}}$ or $\alpha>3$ and $\lambda<\frac{\sqrt{2}(n+2)}{\sqrt{2 n+1}}$. From Eq.(20), we can obtain $\Omega_{\phi}=1$ at point $A$, this means the kinetic dominated solution exists near this point. From Eq.(23), one can obtain $q=2$ (i.e. decelerating phase of the universe) and the average scale factor of the model is evolves as $a \propto t^{\frac{1}{3}}$ at this point A. Using above relation of scale factor with Eqs.(2) and (3), the directional Hubble parameters $H_{1}$ and $H_{2}$ are given by $\frac{n}{(n+2) t}$ and $\frac{1}{(n+2) t}$ respectively. In view of Eq.(22) with $x=1 \& y=0$, the effective equation of state parameter $\gamma_{\phi}$ is equal to one.

Nature of point B, unstable if $\alpha<3$ and $\lambda>-\frac{\sqrt{2}(n+2)}{\sqrt{2 n+1}}$ whereas this point becomes stable if $\alpha>3$ and $\lambda<-\frac{\sqrt{2}(n+2)}{\sqrt{2 n+1}}$. This critical point is saddle if either $\alpha<3$ and $\lambda<-\frac{\sqrt{2}(n+2)}{\sqrt{2 n+1}}$ or $\alpha>3$ and $\lambda>-\frac{\sqrt{2}(n+2)}{\sqrt{2 n+1}}$. From Eq.(20), we can obtain $\Omega_{\phi}=1$ at point $\mathrm{B}$, this means the kinetic dominated solution exists near this point. From Eq.(23), one can obtain $q=2$ (i.e. decelerating phase of the universe) and the average scale factor of the model is evolves as $a \propto t^{\frac{1}{3}}$ at this point B. Using above relation of scale factor with Eqs.(2) and (3), the directional Hubble parameters $H_{1}$ and $H_{2}$ are given by $\frac{n}{(n+2) t}$ and $\frac{1}{(n+2) t}$ respectively. In view of Eq.(22) with $x=-1 \& y=0$, the effective equation of state parameter $\gamma_{\phi}$ is equal to one.

Nature of point $\mathrm{C}$, unstable if $\alpha>3$ and $\lambda<\frac{(3+\alpha)(n+2)}{\sqrt{6 \alpha(2 n+1)}}$ whereas this point becomes stable if $\alpha<3$ and $\lambda>\frac{(3+\alpha)(n+2)}{\sqrt{6 \alpha(2 n+1)}}$. This critical point is saddle if either $\alpha>3$ and $\lambda>\frac{(3+\alpha)(n+2)}{\sqrt{6 \alpha(2 n+1)}}$ or $\alpha<3$ and $\lambda<\frac{(3+\alpha)(n+2)}{\sqrt{6 \alpha(2 n+1)}}$. From Eq.(23), one can obtain there is accelerating phase of the universe near point $\mathrm{C}$ for $\alpha<-1$ and the average scale factor of the model is evolves as $a \propto \frac{(3+\alpha)}{2} t^{\frac{2}{3+\alpha}}$ at this point $\mathrm{C}$. Using above relation of scale factor with Eqs.(2) and (3), the directional Hubble parameters $H_{1}$ and $H_{2}$ are given by $\frac{6 n}{(n+2)(3+\alpha) t}$ and $\frac{6}{(n+2)(3+\alpha) t}$ respectively. In view of Eq.(22), the effective equation of state parameter $\gamma_{\phi}$ is equal to one at point $\mathrm{C}$.

Nature of point $\mathrm{D}$, unstable if $\alpha>3$ and $\lambda>-\frac{(3+\alpha)(n+2)}{\sqrt{6 \alpha(2 n+1)}}$ whereas this point becomes stable if $\alpha<3$ and $\lambda<\frac{(3+\alpha)(n+2)}{\sqrt{6 \alpha(2 n+1)}}$. This critical point is Saddle if either $\alpha>3$ and $\lambda<\frac{(3+\alpha)(n+2)}{\sqrt{6 \alpha(2 n+1)}}$ or $\alpha<3$ and $\lambda>-\frac{(3+\alpha)(n+2)}{\sqrt{6 \alpha(2 n+1)}}$. From Eq.(23), one 
can obtain there is accelerating phase of the universe near point $\mathrm{D}$ for $\alpha<-1$ and the average scale factor of the model is evolves as $a \propto \frac{(3+\alpha)}{2} t^{\frac{2}{3+\alpha}}$ at this point. Using above relation of scale factor with Eqs.(2) and (3), the directional Hubble parameters $H_{1}$ and $H_{2}$ are given by $\frac{6 n}{(n+2)(3+\alpha) t}$ and $\frac{6}{(n+2)(3+\alpha) t}$ respectively. In view of Eq.(22), the effective equation of state parameter $\gamma_{\phi}$ is equal to one at point D.

Nature of point E, unstable if $\lambda^{2}>\frac{2(n+2)^{2}}{(2 n+1)}$ and $\alpha<\frac{3(2 n+1)}{(n+2)^{2}} \lambda^{2}-3$ whereas this point becomes stable if $\lambda^{2}<\frac{2(n+2)^{2}}{(2 n+1)}$ and $\alpha>\frac{3(2 n+1)}{(n+2)^{2}} \lambda^{2}-3$. This critical point is saddle if either $\lambda^{2}>\frac{2(n+2)^{2}}{(2 n+1)}$ and $\alpha>\frac{3(2 n+1)}{(n+2)^{2}} \lambda^{2}-3$ or $\lambda^{2}<\frac{2(n+2)^{2}}{(2 n+1)}$ and $\alpha<\frac{3(2 n+1)}{(n+2)^{2}} \lambda^{2}-3$. From Eq.(23), one can obtain there is accelerating phase of the universe near point $\mathrm{E}$ for $\lambda^{2}<\frac{2(n+2)^{2}}{3(2 n+1)}$ and the average scale factor of the model is evolves as $a \propto \frac{\lambda^{2} l^{\prime 2}}{3} t \frac{3}{\lambda^{2} l^{\prime 2}}$ at this point. Using above relation of scale factor with Eqs.(2) and (3), the directional Hubble parameters $H_{1}$ and $H_{2}$ are given by $\frac{9 n}{(n+2) \lambda^{2} l^{\prime 2} t}$ and $\frac{9}{(n+2) \lambda^{2} l^{\prime 2} t}$ respectively. In view of Eq.(22), the effective equation of state parameter is given by $\gamma_{\phi}=\frac{2 \lambda^{2} l^{\prime 2}}{9}-1$ at point $\mathrm{E}$.

Nature of point $\mathrm{F}$, unstable if $\frac{\left(X_{F}\right)^{1 / 2}}{6 \lambda l^{\prime}+2 \alpha \lambda l^{\prime}}+\frac{3 \alpha^{2}+6 \alpha-9}{12+4 \alpha}-\frac{\alpha \lambda^{2} l^{\prime 3}}{9+3 \alpha}>0$ and $-\frac{\left(X_{F}\right)^{1 / 2}}{6 \lambda l^{\prime}+2 \alpha \lambda l^{\prime}}+$ $\frac{3 \alpha^{2}+6 \alpha-9}{12+4 \alpha}-\frac{\alpha \lambda^{2} l^{\prime 3}}{9+3 \alpha}>0$ whereas this point becomes stable if $\frac{\left(X_{F}\right)^{1 / 2}}{6 \lambda l^{\prime}+2 \alpha \lambda l^{\prime}}+\frac{3 \alpha^{2}+6 \alpha-9}{12+4 \alpha}-$ $\frac{\alpha \lambda^{2} l^{\prime 3}}{9+3 \alpha}<0$ and $-\frac{\left(X_{F}\right)^{1 / 2}}{6 \lambda l^{\prime}+2 \alpha \lambda l^{\prime}}+\frac{3 \alpha^{2}+6 \alpha-9}{12+4 \alpha}-\frac{\alpha \lambda^{2} l^{\prime 3}}{9+3 \alpha}<0$. This critical point is saddle if either $\frac{\left(X_{F}\right)^{1 / 2}}{6 \lambda l^{\prime}+2 \alpha \lambda l^{\prime}}+\frac{3 \alpha^{2}+6 \alpha-9}{12+4 \alpha}-\frac{\alpha \lambda^{2} l^{\prime 3}}{9+3 \alpha}>0$ and $-\frac{\left(X_{F}\right)^{1 / 2}}{6 \lambda l^{\prime}+2 \alpha \lambda l^{\prime}}+\frac{3 \alpha^{2}+6 \alpha-9}{12+4 \alpha}-\frac{\alpha \lambda^{2} l^{\prime 3}}{9+3 \alpha}<0$ or $\frac{\left(X_{F}\right)^{1 / 2}}{6 \lambda l^{\prime}+2 \alpha \lambda l^{\prime}}+\frac{3 \alpha^{2}+6 \alpha-9}{12+4 \alpha}-\frac{\alpha \lambda^{2} l^{\prime 3}}{9+3 \alpha}<0$ and $-\frac{\left(X_{F}\right)^{1 / 2}}{6 \lambda l^{\prime}+2 \alpha \lambda l^{\prime}}+\frac{3 \alpha^{2}+6 \alpha-9}{12+4 \alpha}-\frac{\alpha \lambda^{2} l^{\prime 3}}{9+3 \alpha}>0$. From Eq.(23), one can obtain there is accelerating phase of the universe near point $\mathrm{F}$ for $\alpha<-1 / 3$ and the average scale factor of the model is evolves as $a \propto \frac{(3+\alpha)}{2} t^{\frac{2}{3+\alpha}}$ at this point. Using above relation of scale factor with Eqs.(2) and (3), the directional Hubble parameters $H_{1}$ and $H_{2}$ are given by $\frac{6 n}{(n+2)(3+\alpha) t}$ and $\frac{6}{(n+2)(3+\alpha) t}$ respectively. In view of Eq.(22), the effective equation of state parameter is given by $\gamma_{\phi}=\frac{4 \alpha \lambda^{2} l^{2}}{6(3+\alpha)^{2}-4 \alpha \lambda^{2} l^{2}}$ at point $\mathrm{F}$.

From the point of view of the classical stability for model II, the sound speed for this model is given by

$$
C_{s}^{2}=\frac{6 x^{2}-\frac{6 \sqrt{2} \sqrt{2 n+1}}{n+2} \lambda x y^{2}-\alpha\left(1-x^{2}-y^{2}\right)}{3+3 x^{2}-3 y^{2}}
$$

We have given both local and classical stability for model II in Table 6. It is observed that, when $\alpha<3$, the points A and B are not locally stable if $\lambda<\frac{\sqrt{2}(n+2)}{\sqrt{2 n+1}}$ and $\lambda>-\frac{\sqrt{2}(n+2)}{\sqrt{2 n+1}}$ respectively while these points are classically stable for all $\lambda$ and $\alpha$. It is also observed that when $\alpha>3$, point $\mathrm{C}$ and $\mathrm{D}$ are not unstable when $\lambda<\frac{(3+\alpha)(n+2)}{\sqrt{6 \alpha(2 n+1)}}$ and $\lambda>-\frac{(3+\alpha)(n+2)}{\sqrt{6 \alpha(2 n+1)}}$ respectively while these critical points are 


\begin{tabular}{|c|c|c|c|c|}
\hline Point & $x_{\star}$ & $y_{\star}$ & Local stability & Classical stability \\
\hline$\overline{\mathrm{A}}$ & 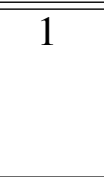 & 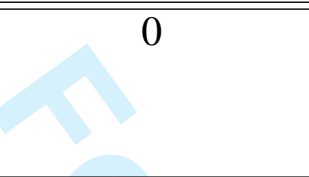 & $\begin{array}{c}\text { Stable point if } \alpha>3 \\
\text { and } \lambda>\frac{\sqrt{2}(n+2)}{\sqrt{2 n+1}} \\
\text { otherwise unstable }\end{array}$ & Stable \\
\hline B & -1 & 0 & $\begin{array}{c}\text { Stable point if } \alpha>3 \\
\text { and } \lambda<-\frac{\sqrt{2}(n+2)}{\sqrt{2 n+1}} \\
\text { otherwise unstable }\end{array}$ & Stable \\
\hline $\mathrm{C}$ & $\frac{\sqrt{\alpha}}{\sqrt{3}}$ & 0 & $\begin{array}{c}\text { Stable if } \alpha<3 \\
\text { and } \lambda>\frac{(3+\alpha)(n+2)}{\sqrt{6 \alpha(2 n+1)}} \\
\text { otherwise unstable }\end{array}$ & $\begin{array}{c}\text { Stable if } \\
0<\frac{\alpha}{3}<1\end{array}$ \\
\hline $\mathrm{D}$ & $-\frac{\sqrt{\alpha}}{\sqrt{3}}$ & 0 & $\begin{array}{c}\text { Stable if } \alpha<3 \\
\lambda<\frac{(3+\alpha)(n+2)}{\sqrt{6 \alpha(2 n+1)}} \\
\text { otherwise unstable }\end{array}$ & $\begin{array}{c}\text { Stable if } \\
0<\frac{\alpha}{3}<1\end{array}$ \\
\hline $\mathrm{E}$ & $\frac{\lambda l^{\prime}}{3}$ & $\left(1-\left(\frac{\lambda l^{\prime}}{3}\right)^{2}\right)^{1 / 2}$ & $\begin{array}{c}\text { Stable if } \lambda^{2}<\frac{2(n+2)^{2}}{(2 n+1)} \\
\text { and } \alpha>\frac{3(2 n+1)}{(n+2)^{2}} \lambda^{2}-3 \\
\text { otherwise unstable }\end{array}$ & $\begin{array}{c}\text { Stable if } \\
\lambda<\frac{\sqrt{2}(n+2)}{\sqrt{2 n+1}}\end{array}$ \\
\hline$F$ & $\frac{\alpha+3}{2 \lambda l^{\prime}}$ & $\frac{\left((\alpha+3)^{2}-\frac{4 \alpha \lambda^{2} l^{\prime 2}}{3}\right)^{1 / 2}}{2 \lambda l^{\prime}}$ & $\begin{array}{c}\begin{array}{c}\text { Stable if } \\
\frac{\left(X_{F}\right)^{1 / 2}}{6 \lambda l^{\prime}+2 \alpha \lambda l^{\prime}}+\frac{3 \alpha^{2}+6 \alpha-9}{12+4 \alpha}-\frac{\alpha \lambda^{2} l^{\prime 3}}{9+3 \alpha}<0 \\
\text { and }\end{array} \\
-\frac{\left(X_{F}\right)^{1 / 2}}{6 \lambda l^{\prime}+2 \alpha \lambda l^{\prime}}+\frac{3 \alpha^{2}+6 \alpha-9}{12+4 \alpha}-\frac{\alpha \lambda^{2} l^{\prime 3}}{9+3 \alpha}<0 \\
\text { otherwise unstable }\end{array}$ & $\begin{array}{c}\text { Stable if } \\
0<\frac{\alpha}{3}<1\end{array}$ \\
\hline
\end{tabular}

Table 6: Local and classical stability criteria at fixed points for the model-II. 
classically stable under some restrictions on $\alpha$ i.e. $0<\alpha<3$. From Table 6 , it is noted that the point $\mathrm{E}$ is stable when $\lambda^{2}<\frac{2(n+2)^{2}}{(2 n+1)}$ and $\alpha>\frac{3(2 n+1)}{(n+2)^{2}} \lambda^{2}-3$ whereas this point corresponds to classical stability when $\lambda<\frac{\sqrt{2}(n+2)}{\sqrt{2 n+1}}$. It is interesting that the point $\mathrm{F}$ is locally and classically stable under some restrictions on $\lambda$ and $\alpha$.

\section{Coupling Model (III): $Q=\Gamma \rho_{\gamma}$}

Here $\Gamma$ is a constant. When $\Gamma>0$, the energy transfer corresponds to decay of from dark matter to dark energy whereas when $\Gamma<0$, a transfer of energy from dark energy to dark matter.

In this Section, we are considering the dark sector interaction as $Q=\Gamma \rho_{\gamma}$ for LRS BI cosmological model, while still keeping the same variables $x$ and $y$ as mentioned in Sec. 3. Here we introduce a new variable $z$, chosen so as to maintain compactness of the phase space.

$$
z=\frac{H_{0}}{H+H_{0}}
$$

where $0 \leq z \leq 1$. When $z \rightarrow 0$ corresponds to $H \rightarrow \infty$ then critical point is for the early universe whereas for the late universe $z \rightarrow 1$ corresponds $H \rightarrow 0$.

We also rescale to a dimensionless coupling constant

$$
\gamma_{\star}=\frac{\Gamma}{H_{0}}
$$

The autonomous system of equations for model III are

$$
\begin{gathered}
\frac{d x}{d N} \equiv x^{\prime}=-3 x+\frac{3 \sqrt{(2 n+1)}}{\sqrt{2}(n+2)} \lambda y^{2}+\frac{3}{2} x\left(1+x^{2}-y^{2}\right)-\frac{\gamma_{\star}\left(1-x^{2}-y^{2}\right) z}{2 x(z-1)} \\
\frac{d y}{d N} \equiv y^{\prime}=-\lambda \frac{3 \sqrt{(2 n+1)}}{\sqrt{2}(n+2)} x y+\frac{3}{2} y\left(1+x^{2}-y^{2}\right) \\
\frac{d z}{d N} \equiv z^{\prime}=\frac{3}{2} z(1-z)\left(1+x^{2}-y^{2}\right)
\end{gathered}
$$

\subsection{Phase-space analysis of the model III}

From Eqs. (32,33\& 34), the autonomous system of equations have seven critical points namely A,B,C,D,E,F and G:

- Point A $(1,0,0)$ 


\begin{tabular}{|c|c|c|c|c|c|c|c|}
\hline Point & $x_{\star}$ & $y_{\star}$ & $z_{\star}$ & $\Omega_{\phi}$ & $\gamma_{\phi}$ & $q$ & Existence \\
\hline \hline A & 1 & 0 & 0 & 1 & 1 & 2 & $\forall \lambda, \gamma$ \\
\hline B & -1 & 0 & 0 & 1 & 1 & 2 & $\forall \lambda, \gamma$ \\
\hline C & $\frac{\lambda l^{\prime}}{3}$ & $\left(1-\frac{\lambda^{2} l^{\prime 2}}{9}\right)^{1 / 2}$ & 0 & 1 & $-1+\frac{2 \lambda^{2} l^{\prime 2}}{9}$ & $-1+\frac{\lambda^{2} l^{\prime 2}}{3}$ & $\lambda \leq \frac{\sqrt{2}(n+2)}{\sqrt{2 n+1}}$ \\
\hline D & $\frac{3}{2 \lambda l^{\prime}}$ & $\frac{3}{2 \lambda l^{\prime}}$ & 0 & $\frac{3}{\lambda l^{\prime}}$ & 0 & $\frac{1}{2}$ & $\lambda \geq \frac{\sqrt{2}(n+2)}{\sqrt{2 n+1}}$ \\
\hline E & 1 & 0 & 1 & 1 & 1 & 2 & $\forall \lambda, \gamma$ \\
\hline F & -1 & 0 & 1 & 1 & 1 & 2 & $\forall \lambda, \gamma$ \\
\hline G & $\frac{\lambda l^{\prime}}{3}$ & $\left(1-\frac{\lambda^{2} l^{\prime 2}}{9}\right)^{1 / 2}$ & 1 & 1 & $-1+\frac{2 \lambda^{2} l^{\prime 2}}{9}$ & $-1+\frac{\lambda^{2} l^{\prime 2}}{3}$ & $\lambda \leq \frac{\sqrt{2}(n+2)}{\sqrt{2 n+1}}$ \\
\hline
\end{tabular}

Table 7: Physical parameters at fixed points for model III.

\begin{tabular}{|c|c|c|c|c|c|c|}
\hline Point & $x_{\star}$ & $y_{\star}$ & $z_{\star}$ & $\lambda_{1}$ & $\lambda_{2}$ & $\lambda_{3}$ \\
\hline \hline $\mathrm{A}$ & 1 & 0 & 0 & 3 & 3 & $3-\lambda l^{\prime}$ \\
\hline $\mathrm{B}$ & -1 & 0 & 0 & 3 & 3 & $3+\lambda l^{\prime}$ \\
\hline $\mathrm{C}$ & $\frac{\lambda l^{\prime}}{3}$ & $\left(1-\frac{\lambda^{2} l^{\prime 2}}{9}\right)^{1 / 2}$ & 0 & $\frac{\lambda^{2} l^{\prime 2}}{3}$ & $-3+\frac{2 \lambda^{2} l^{\prime 2}}{3}$ & $-3+\frac{\lambda^{2} l^{\prime 2}}{3}$ \\
\hline $\mathrm{D}$ & $\frac{3}{2 \lambda l^{\prime}}$ & $\frac{3}{2 \lambda l^{\prime}}$ & 0 & $\frac{3}{2}$ & $-\frac{3}{4}+\frac{\left(\frac{81}{4}-\frac{63 \lambda^{2} l^{\prime 2}}{16}\right)^{1 / 2}}{3 l^{\prime}}$ & $-\frac{3}{4}-\frac{\left(\frac{81}{4}-\frac{63 \lambda^{2} l^{\prime 2}}{16}\right)^{1 / 2}}{\lambda l^{\prime}}$ \\
\hline $\mathrm{E}$ & 1 & 0 & 1 & -3 & $3-\lambda l^{\prime}$ & $-\operatorname{sgn}\left(\gamma_{\star}\right) \infty$ \\
\hline $\mathrm{F}$ & -1 & 0 & 1 & -3 & $3-\lambda l^{\prime}$ & $-\operatorname{sgn}\left(\gamma_{\star}\right) \infty$ \\
\hline $\mathrm{G}$ & $\frac{\lambda l^{\prime}}{3}$ & $\left(1-\frac{\lambda^{2} l^{\prime 2}}{9}\right)^{1 / 2}$ & 1 & $-\frac{\lambda^{2} l^{\prime 2}}{3}$ & $-3+\frac{\lambda^{2} l^{\prime 2}}{3}$ & $-\operatorname{sgn}\left(\gamma_{\star}\right) \infty$ \\
\hline
\end{tabular}

Table 8: Critical points and eigenvalues for model III.

- Point B $(-1,0,0)$

- Point C $\left(\frac{l^{\prime} \lambda}{3},\left(1-\left(\frac{l^{\prime} \lambda}{3}\right)^{2}\right)^{1 / 2}, 0\right)$

- Point D $\left(\frac{3}{2 l^{\prime} \lambda}, \frac{3}{2 l^{\prime} \lambda}, 0\right)$

- Point E $(1,0,1)$

- Point F $(-1,0,1)$

- Point $\mathrm{G}\left(\frac{l^{\prime} \lambda}{3},\left(1-\left(\frac{l^{\prime} \lambda}{3}\right)^{2}\right)^{1 / 2}, 1\right)$

Here it is noted that from Table 7, the critical points A,B,E and $\mathrm{F}$ are always exist for all values of $\lambda$ and $\gamma$, while the critical points $\mathrm{C}$ and $\mathrm{G}$ exist when $\lambda \leq$ $\frac{\sqrt{2}(n+2)}{\sqrt{2 n+1}}$. On the other hand critical point $\mathrm{D}$ exists when $\lambda \geq \frac{\sqrt{2}(n+2)}{\sqrt{2 n+1}}$. The nature of critical points and eigenvalues of the jacobian matrix at critical points are given in Table 8. 


\subsection{Local and classical stability analysis of model III}

In this section, we are discussing the stability of the critical points A,B,C, D, E,F and $\mathrm{G}$ of the autonomous system of Eqs.(32, 33 \& 34).

Nature of point A, unstable if $\lambda<\frac{\sqrt{2}(n+2)}{\sqrt{2 n+1}}$ whereas this point becomes saddle if $\lambda>\frac{\sqrt{2}(n+2)}{\sqrt{2 n+1}}$. From Eq.(20), we can obtain $\Omega_{\phi}=1$ at point A, this means the kinetic dominated solution exists near this point. From Eq.(23), one can obtain $q=2$ (i.e. decelerating phase of the universe) and the average scale factor of the model is evolves as $a \propto t^{\frac{1}{3}}$ at this point A. Using above relation of scale factor with Eqs.(2) and (3), the directional Hubble parameters $H_{1}$ and $H_{2}$ are given by $\frac{n}{(n+2) t}$ and $\frac{1}{(n+2) t}$ respectively. In view of Eq.(22) with $x=1, y=0 \& z=0$, the effective equation of state parameter $\gamma_{\phi}$ is equal to one.

Nature of point $\mathrm{B}$, unstable if $\lambda<\frac{\sqrt{2}(n+2)}{\sqrt{2 n+1}}$ whereas this point becomes saddle if $\lambda>\frac{\sqrt{2}(n+2)}{\sqrt{2 n+1}}$. From Eq.(20), we can obtain $\Omega_{\phi}=1$ at point B, this means the kinetic dominated solution exists near this point. From Eq.(23), one can obtain $q=2$ (i.e. decelerating phase of the universe) and the average scale factor of the model is evolves as $a \propto t^{\frac{1}{3}}$ at this point B. Using above relation of scale factor with Eqs.(2) and (3), the directional Hubble parameters $H_{1}$ and $H_{2}$ are given by $\frac{n}{(n+2) t}$ and $\frac{1}{(n+2) t}$ respectively. In view of Eq.(22) with $x=-1, y=0 \& z=0$ the effective equation of state parameter $\gamma_{\phi}$ is equal to one.

Nature of point $C$ is saddle if $\lambda<\frac{\sqrt{2}(n+2)}{\sqrt{2 n+1}}$. From Eq.(23), one can obtain there is accelerating phase of the universe near point $\mathrm{C}$ for $\lambda^{2}<\frac{2(n+2)^{2}}{3(2 n+1)}$ and the average scale factor of the model is evolves as $a \propto \frac{\lambda^{2} l^{2}}{3} t \frac{3}{\lambda^{2} l^{\prime 2}}$ at this point C. Using above relation of scale factor with Eqs.(2) and (3), the directional Hubble parameters $H_{1}$ and $H_{2}$ are given by $\frac{9 n}{(n+2) \lambda^{2} l^{\prime 2} t}$ and $\frac{9}{(n+2) \lambda^{2} l^{\prime 2} t}$ respectively. In view of Eq.(22), the effective equation of state parameter is $\gamma_{\phi}=\frac{(2 n+1)}{(n+2)} \lambda^{2}-1$ at point $\mathrm{C}$.

Nature of point $\mathrm{D}$ is saddle node for $\frac{8(n+2)}{7(2 n+1)}>\lambda^{2}>\frac{n+2}{3(2 n+1)}$ and saddle focus for $\frac{36}{7}<\lambda^{2} l^{\prime 2}$. From Eq.(20), we can obtain $\Omega_{\phi}=1$ at point $\mathrm{D}$, this means the kinetic dominated solution exists near this point. From Eq.(23), one can obtain $q=1 / 2$ (i.e. decelerating phase of the universe) and the average scale factor of the model is evolves as $a \propto t^{\frac{2}{3}}$ at this point. Using above relation of scale factor with Eqs.(2) and (3), the directional Hubble parameters $H_{1}$ and $H_{2}$ are given by $\frac{2 n}{(n+2) t}$ and $\frac{2}{(n+2) t}$ respectively. In view of Eq.(22), the effective equation of state parameter $\gamma_{\phi}$ is zero at point $\mathrm{D}$.

Nature of point $\mathrm{E}$ is stable node for $\lambda>\frac{\sqrt{2}(n+2)}{\sqrt{2 n+1}}$ and $\gamma_{*}>0$ whereas this point becomes saddle node if either $\lambda>\frac{\sqrt{2}(n+2)}{\sqrt{2 n+1}}$ and $\gamma_{*}<0$ or $\lambda<\frac{\sqrt{2}(n+2)}{\sqrt{2 n+1}}$ and all $\gamma_{*}$. From Eq.(20), we can obtain $\Omega_{\phi}=1$ at point E, this means the kinetic 
dominated solution exists near this point. From Eq.(23), one can obtain $q=2$ (i.e. decelerating phase of the universe) and the average scale factor of the model is evolves as $a \propto t^{\frac{1}{3}}$ at this point. Using above relation of scale factor with Eqs.(2) and (3), the directional Hubble parameters $H_{1}$ and $H_{2}$ are given by $\frac{n}{(n+2) t}$ and $\frac{1}{(n+2) t}$ respectively. In view of Eq.(22), the effective equation of state parameter $\gamma_{\phi}$ is equal to one at point $\mathrm{E}$.

Nature of point $\mathrm{F}$ is stable node for $\lambda<-\frac{\sqrt{2}(n+2)}{\sqrt{2 n+1}}$ and $\gamma_{*}>0$ whereas saddle node if either $\lambda<-\frac{\sqrt{2}(n+2)}{\sqrt{2 n+1}}$ and $\gamma_{*}<0$ or $\lambda>\frac{\sqrt{2}(n+2)}{\sqrt{2 n+1}}$ and all $\gamma_{*}$. From Eq.(20), we can obtain $\Omega_{\phi}=1$ at point $F$, this means the kinetic dominated solution exists near this point. From Eq.(23), one can obtain $q=2$ (i.e. decelerating phase of the universe) and the average scale factor of the model is evolves as $a \propto t^{\frac{1}{3}}$ at this point. Using above relation of scale factor with Eqs.(2) and (3), the directional Hubble parameters $H_{1}$ and $H_{2}$ are given by $\frac{n}{(n+2) t}$ and $\frac{1}{(n+2) t}$ respectively. In view of Eq.(22), the effective equation of state parameter $\gamma_{\phi}$ is equal to one at point $\mathrm{F}$.

Nature of point G is stable node for $\gamma_{*}>0$ and saddle node $\gamma_{*}<0$. From Eq.(23), one can obtain there is accelerating phase of the universe near point $\mathrm{G}$ for $\lambda^{2}<\frac{2(n+2)^{2}}{3(2 n+1)}$ and the average scale factor of the model is evolves as $a \propto \frac{\lambda^{2} l^{\prime 2}}{3} t \frac{3}{\lambda^{2} l^{\prime 2}}$ at this point. Using above relation of scale factor with Eqs.(2) and (3), the directional Hubble parameters $H_{1}$ and $H_{2}$ are given by $\frac{9 n}{(n+2) \lambda^{2} l^{\prime 2} t}$ and $\frac{9}{(n+2) \lambda^{2} l^{\prime 2} t}$ respectively. In view of Eq.(22), the effective equation of state parameter is given by $\gamma_{\phi}=\frac{2 \lambda^{2} l^{\prime 2}}{9}-1$ at point $\mathrm{G}$.

From the point of view of the classical stability of model III, the sound speed for this model is given by

$$
C_{s}^{2}=\frac{6 x^{2}-\frac{6 \sqrt{2} \sqrt{2 n+1}}{n+2} \lambda x y^{2}-\gamma_{*} \frac{z}{1-z}\left(1-x^{2}-y^{2}\right)}{3+3 x^{2}-3 y^{2}}
$$

We have given both local and classical stability for model III in Table 9. It is observed that, when $\alpha<3$, points $\mathrm{A}$ and $\mathrm{B}$ are not locally stable when $\lambda<$ $\frac{\sqrt{2}(n+2)}{\sqrt{2 n+1}}$ and $\lambda>-\frac{\sqrt{2}(n+2)}{\sqrt{2 n+1}}$ respectively while these critical points are classically stable for all $\lambda$. When $\lambda<\frac{\sqrt{2}(n+2)}{\sqrt{2 n+1}}$, the point $\mathrm{C}$ is locally and classically stable both while point $\mathrm{D}$ is saddle node when $\frac{8(n+2)}{7(2 n+1)}>\lambda^{2}>\frac{n+2}{3(2 n+1)}$ and classically stable for all $\lambda$. From Table 9, It is also observed that points $\mathrm{E}$ and $\mathrm{F}$ are not stable node when $\lambda>\frac{\sqrt{2}(n+2)}{\sqrt{2 n+1}}$ and $\lambda<-\frac{\sqrt{2}(n+2)}{\sqrt{2 n+1}}$ respectively. It is interesting that the critical point $\mathrm{G}$ is locally stable when $\gamma_{\star}>0$ but classically stability is undefined due to the limit $z_{\star} \rightarrow 1$. 


\begin{tabular}{|c|c|c|c|c|c|}
\hline Point & $x_{\star}$ & $y_{\star}$ & $z_{\star}$ & Local stability & Classical stability \\
\hline $\mathrm{A}$ & 1 & 0 & 0 & $\begin{array}{l}\text { Unstable if } \lambda<\frac{\sqrt{2}(n+2)}{\sqrt{2 n+1}} \\
\text { Saddle if } \lambda>\frac{\sqrt{2}(n+2)}{\sqrt{2 n+1}}\end{array}$ & Stable \\
\hline B & -1 & 0 & 0 & $\begin{array}{l}\text { Unstable if } \lambda>-\frac{\sqrt{2}(n+2)}{\sqrt{2 n+1}} \\
\text { Saddle if } \lambda<-\frac{\sqrt{2(n+2)}}{\sqrt{2 n+1}}\end{array}$ & Stable \\
\hline $\mathrm{C}$ & $\frac{\lambda l^{\prime}}{3}$ & $\left(1-\frac{\lambda^{2} l^{\prime 2}}{9}\right)^{1 / 2}$ & 0 & $\begin{array}{l}\text { Saddle node if } \\
\qquad \lambda<\frac{\sqrt{2}(n+2)}{\sqrt{2 n+1}}\end{array}$ & $\begin{array}{c}\text { Stable if } \\
\lambda<\frac{\sqrt{2}(n+2)}{\sqrt{2 n+1}}\end{array}$ \\
\hline $\mathrm{D}$ & $\frac{3}{2 \lambda l^{\prime}}$ & $\frac{3}{2 \lambda l^{\prime}}$ & 0 & $\begin{array}{c}\text { Saddle node for } \\
\frac{36}{7}>\lambda^{2} l^{\prime 2}>\frac{3}{2} \\
\text { saddle focus for } \\
\frac{36}{7}<\lambda^{2} l^{\prime 2}\end{array}$ & Stable \\
\hline $\mathrm{E}$ & 1 & 0 & 1 & $\begin{array}{c}\text { Stable node for } \lambda>\frac{\sqrt{2}(n+2)}{\sqrt{2 n+1}} \& \gamma_{*}>0 \\
\text { saddle node if either } \lambda>\frac{\sqrt{2}(n+2)}{\sqrt{2 n+1}} \& \gamma_{*}<0 \\
\text { or } \lambda<\frac{\sqrt{2}(n+2)}{\sqrt{2 n+1}} \& \text { for all } \gamma_{*}\end{array}$ & Undefined \\
\hline $\mathrm{F}$ & -1 & 0 & 1 & $\begin{array}{c}\text { Stable node for } \lambda<-\frac{\sqrt{2}(n+2)}{\sqrt{2 n+1}} \& \gamma_{*}>0 \\
\text { saddle node if either } \lambda>-\frac{\sqrt{2}(n+2)}{\sqrt{2 n+1}} ; \gamma_{*}<0 \\
\text { or } \lambda>-\frac{\sqrt{2}(n+2)}{\sqrt{2 n+1}} \& \text { for all } \gamma_{*}\end{array}$ & Undefined \\
\hline G & $\frac{\lambda l^{\prime}}{3}$ & $\left(1-\frac{\lambda^{2} l^{\prime 2}}{9}\right)^{1 / 2}$ & 1 & $\begin{array}{l}\text { Stable node for } \gamma_{*}>0 \\
\text { Saddle node for } \gamma_{*}<0\end{array}$ & Undefined \\
\hline
\end{tabular}

Table 9: Local and classical stability criteria at fixed points for the model-III. 


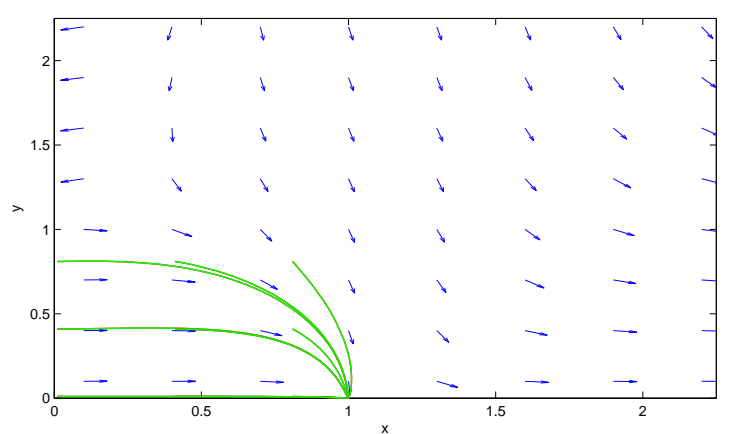

Figure 7: Phase plot of the system (model-II) when $n=0.001, \lambda=4, \alpha=4$,

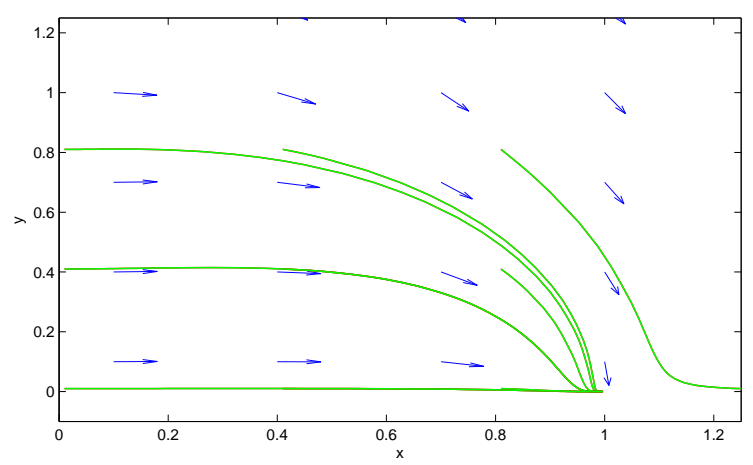

Figure 8: Phase plot of the system (model-II) when $n=1, \lambda=4, \alpha=3.1$,

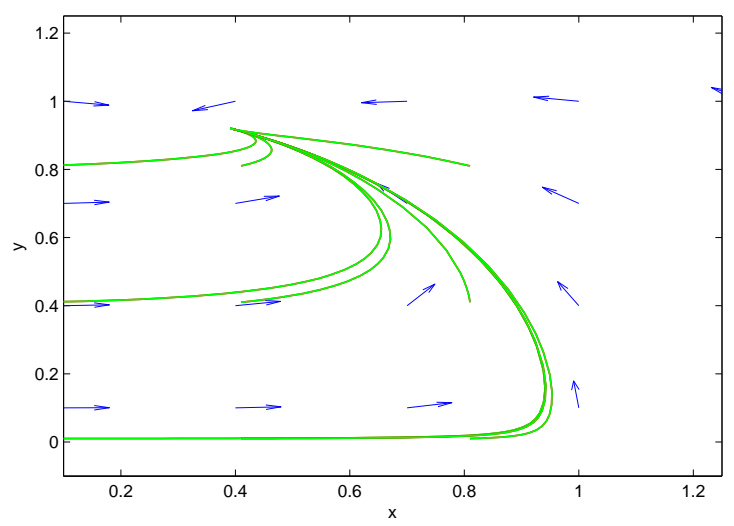

Figure 9: Phase plot of the system (model-II) when $n=100, \lambda=4, \alpha=3.5$, 


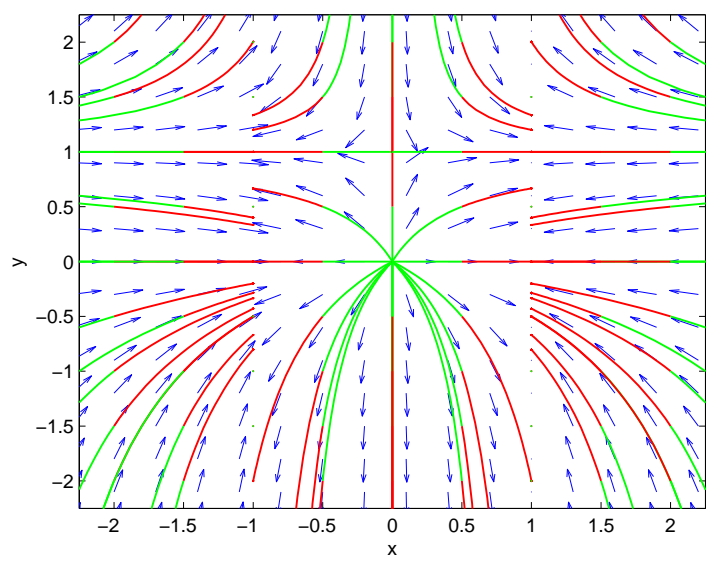

Figure 10: Projection phase plot in $\mathrm{YZ}$ plane

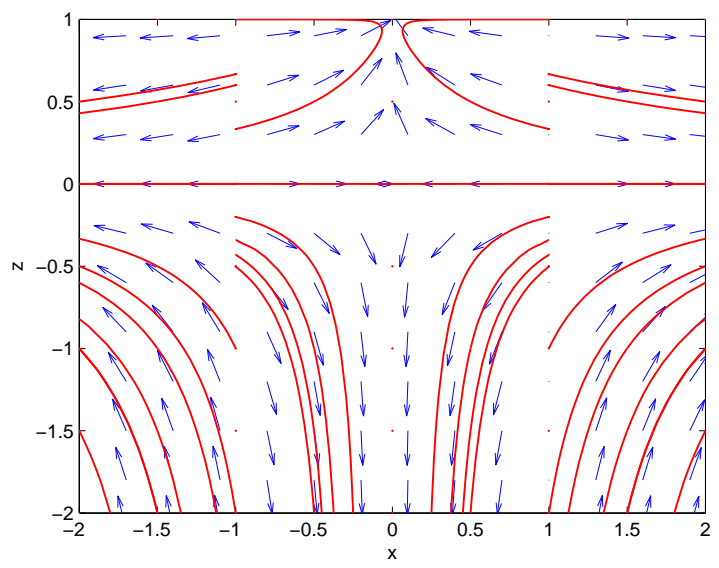

Figure 11: Projection phase plot in XZ plane when $\gamma_{\star}=0.001$

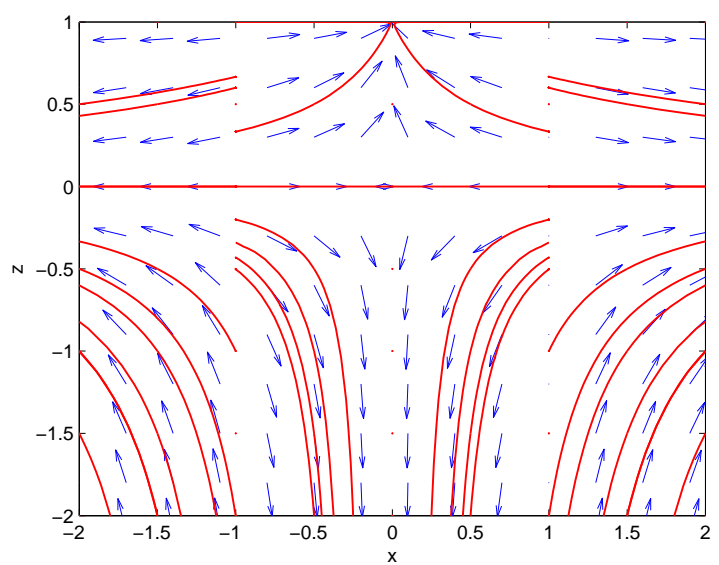

Figure 12: Projection phase plot in XZ plane when $\gamma_{\star}=0.00001$ 24 


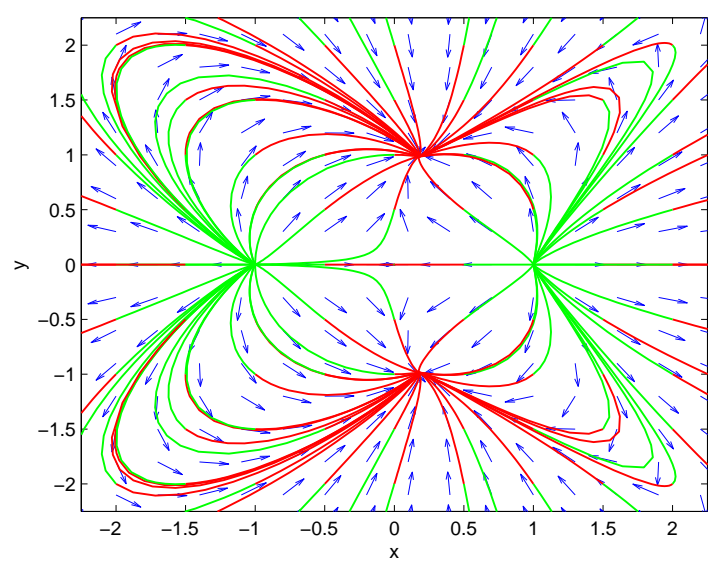

Figure 13: Projection phase plot in XY plane when $n=0.001, \lambda=0.5$

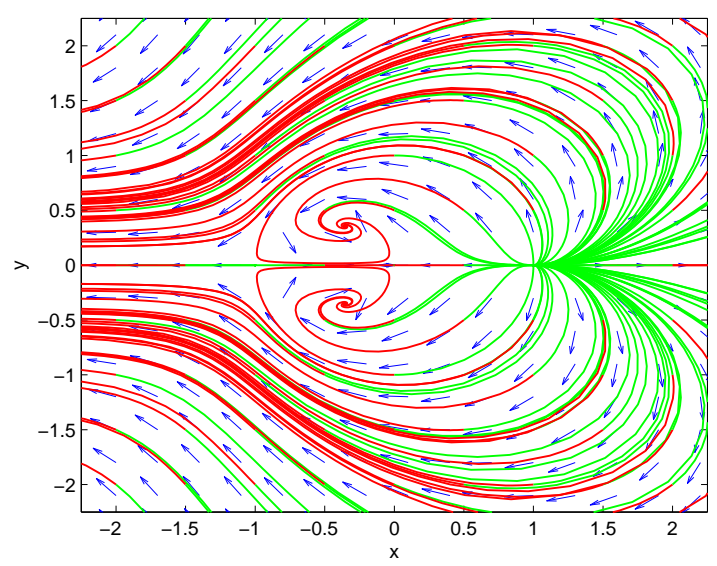

Figure 14: Projection phase plot in XY plane when $n=0.001, \lambda=-4$

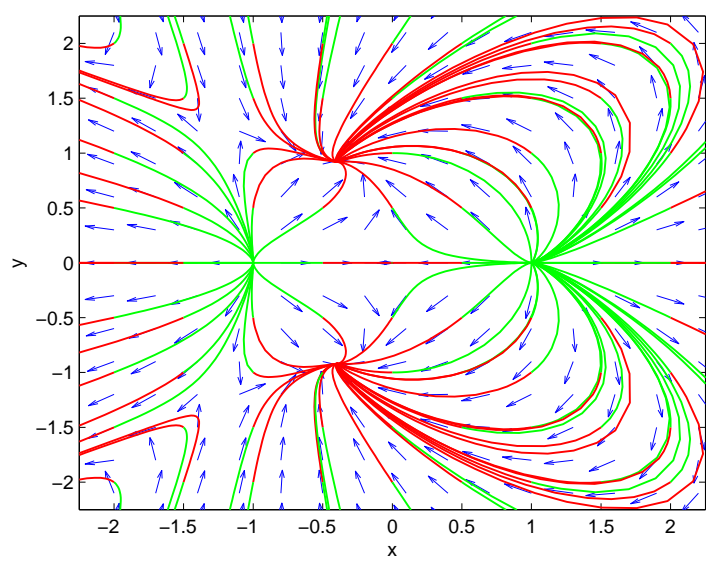

Figure 15: Projection phase plot in XY plane when $n=100, \lambda=-4$ 25 


\section{Conclusions}

This paper dealt the qualitative study of the LRS Bianchi I cosmological model under the background of a universe dominated by dark energy (in the form of exponential quintessence) and cold dark matter, where the energy exchange in the dark sector is considered in three physically viable models as (i) $Q=\sqrt{\frac{2}{3}} \kappa \beta \rho_{\gamma} \dot{\phi}$, (ii) $Q=\alpha H \rho_{\gamma}$ and (iii) $Q=\Gamma \rho_{\gamma}$. We have calculated the critical points and their stability in terms of the parameter $n$. It is interesting to note here that our model is reduced into Bohmer model [50] when $n=1$. We have examined all critical points and discussed their relevant physical parameters near each critical points in three different choices of dark sector coupling $Q$. In comparison to the model with last model there are two new fixed points. In model-I, it is observed that the critical points $\mathrm{A}, \mathrm{B}$ and $\mathrm{C}$ are unstable in local stability analysis while stable in classical stability analysis, whereas other two critical points $\mathrm{D}$ and $\mathrm{E}$ are local and classical stable under some restrictions on $\lambda$ and $\beta$. On the other hand, the critical points $A, B, C$ and $D$ are unstable in local stability analysis while stable in classical stability analysis, whereas other two critical points $\mathrm{E}$ and $\mathrm{F}$ are local and classical stable under some restrictions on $\lambda$ and $\alpha$. In the last model-III, it is observed that critical points A,B,C and D are locally unstable and classically stable whereas critical points $E$ and $F$ are stable node and critical point $G$ is stable when $\gamma_{\star}>0$ but classically undefined due to the limit $z_{\star} \rightarrow 1$. When $\lambda<\frac{\sqrt{2}(n+2)}{\sqrt{2 n+1}}$, the critical point $\mathrm{B}$ is unstable and point $\mathrm{C}$ is stable. As $\lambda$ approaches to $\frac{\sqrt{2}(n+2)}{\sqrt{2 n+1}}$, these two critical points B and C merge into single-fixed point. When $\lambda>\frac{\sqrt{2}(n+2)}{\sqrt{2 n+1}}$ (i.e. on the other side of bifurcation point) the behaviour of these critical points are interchanged. It is interesting to note here that there is a clear bifurcation in the system where $\lambda$ is the bifurcation parameter and $\lambda=\frac{\sqrt{2}(n+2)}{\sqrt{2 n+1}}$ is the bifurcation point. It is worth noting that the recently observed accelerated expansion of the universe near the critical points E (in model-I), F (in model-II) and G (in modelIII) can seen from the autonomous system in the quintessence scalar field with dark sector coupling.

\section{Acknowledgement}

The authors express their sincere thanks to referee for his valuable comments and suggestions. The authors express their sincere thanks to C.S.I.R., New Delhi for the financial assistance under project No. 25(6411)17. 


\section{References}

[1] S. Perlmutter, G. Aldering, M. della Valle, et al.: Nature, 391, 51 (1998). 2

[2] S. Perlmutter, G. Aldering, G. Goldhaber, et al.: ApJ, 517, 565 (1999). 2

[3] S. Perlmutter, S. Gabi, G. Goldhaber, et al.: ApJ, 483, 565 (1997). 2

[4] A. G. Riess, A. V. Filippenko, P. Challis, et al.: AJ, 116, 1009 (1998). 2

[5] P. M. Garnavich, R. P. Kirshner, P. Challis, et al.: ApJL, 493, (1998). L53 2

[6] B. P. Schmidt, N. B. Suntzeff, M. M. Phillips, et al.: ApJ, 507, 46 (1998). 2

[7] J. L. Tonry, B. P. Schmidt, B. Barris, et al.: ApJ, 594, 1 (2003). 2

[8] A. Clocchiatti, B. P. Schmidt, A. V. Filippenko, et al.: ApJ, 642, 1 (2006). 2

[9] P. de Bernardis, P. A. R. Ade, J. J. Bock, et al.: Nature, 404, 955 (2000). 2

[10] S. Hanany, P. Ade, A. Balbi, et al.: ApJL, 545, L5 (2000). 2

[11] D. N. Spergel, L. Verde, H. V. Peiris, et al.: ApJS, 148, 175 (2003). 2

[12] M. Tegmark, M. A. Strauss, M. R. Blanton, et al.: Phys. Rev. D, 69, 103501 (2004). 2

[13] U. Seljak, A. Makarov, P. McDonald, et al.: Phys. Rev. D, 71, 103515 (2005). 2

[14] J. K. Adelman-McCarthy, M. A. Agüeros, S. S. Allam, et al.: ApJS, 162, 38 (2006). 2

[15] C. L. Bennett, , Halpern, M., Hinshaw, G., et al.: ApJS, 148, 1 (2003). 2

[16] S. W. Allen, R. W. Schmidt, H. Ebeling, A. C. Fabian, \& L. van Speybroeck: MNRAS, 353, 457 (2004). 2

[17] A.R. Liddle \& D.H. Lyth: Cosmological inflation and Large Scale Structure. Cambridge University Press, Cambridge, England (2003). 2

[18] J. Magana \& T. Matos: J. Phys. Conf. Ser. 378, 012012 (2012). 2

[19] W. Zimdahl, \& D. Pavón: General Relativity and Gravitation, 36, 1483 (2004). 2 
[20] W. Zimdahl, D. Pavón, L. P. Chimento, \& A. S. Jakubi: in The Tenth Marcel Grossmann Meeting. On recent developments in theoretical and experimental general relativity, gravitation and relativistic field theories, eds. M. Novello, S. Perez Bergliaffa, \& R. Ruffini, 1794 (2005). 2

[21] D. Pavón, S. Sen, \& W. Zimdahl: J. Cosmol. Astropart. Phys., 5, 9 (2004). 2

[22] S. Tsujikawa \& M. Sami: Physics Letters B, 603, 113 (2004). 2

[23] B. Gumjudpai, T. Naskar, M. Sami \& S. Tsujikawa: J. Cosmol. Astropart. Phys., 6, 7 (2005). 2

[24] J. D. Barrow \& T. Clifton: Phys. Rev. D, 73, 103520 (2006). 2

[25] L. Amendola \& S. Tsujikawa: Dark Energy: Theory and Observations by Luca Amendola and Shinji Tsujikawa. Cambridge University Press, (2010). 2

[26] H. Wei: Physics Letters B, 691, 173 (2010). 2

[27] W. Zimdahl, D. Pavón \& L. P. Chimento: Physics Letters B, 521, 133 (2001). 2

[28] Z.K. Guo, N. Ohta \& S. Tsujikawa: Phys. Rev. D, 76, 023508 (2007). 2

[29] G.C. Cabral, R. Maartens \& L.A. Lopez: Phys. Rev. D, 79, 063518 (2009). 2

[30] G.F.R. Ellis \& H. van Elst: in NATO ASIC Proc. 541: Theoretical and Observational Cosmology, eds. M. Lachièze-Rey, arxiv: gr-qc/9812046.(1999). 2

[31] G.F.R. Ellis: Exact and inexact solutions of the Einstein field equations, in The Renaissance of General Relativity and Cosmology, eds. by G. Ellis, A. Lanza, \& J. Miller, 20 (1993). 2

[32] E.W. Kolb \& M.S. Turner: The Early Universe, Addison-Wesley (1990). 2

[33] C.W. Misner: ApJ, 151, 431 (1968). 2

[34] C.W. Misner, K. S. Thorne \& J.A. Wheeler: Gravitation (W.H. Freeman: New York) (1973). 2

[35] B.L. Hu \& L. Parker: Phys. Rev. D, 17, 933 (1978). 2 
[36] S.W. Hawking \& G.F.R. Ellis: The Large Scale Structure of Space-Time, Cambridge University Press, U.K. (1973). 2

[37] V.A. Belinkskii \& I.M. Khalatnikov: Soviet Physics JETP 42, 205 (1976). 2

[38] M.A.H. MacCallum: Anisotropic and inhomogeneous relativistic cosmologies, in General Relativity: An Einstein Centenary Survey, eds. S.W. Hawking \& W. Israel, Cambridge University Press, U.K. (Chapter 11) (1979). 2

[39] G.F.R. Ellis \& R.M. Williams: Flat and Curved Space-Times, Clarendon Press (2000). 2

[40] G.F.R. Ellis \& J. Wainwright: Dynamical Systems in Cosmology, Cambridge Univ. Press (2005). 2

[41] V.A. Belinskii, I.M. Khalatnikov \& E.M. Lifshits: Advances in Physics, 19, 525 (1970). 2

[42] A.A. Coley \& K.A. Dunn: J. Math. Phys. 33, 1772 (1992). 2

[43] A. Burd \& A. Coley: Class. Quant. Grav. 11, 83 (1994). 2

[44] M. Goliath \& G.F.R. Ellis: Phys. Rev. D, 60, 023502 (1999). 2

[45] C.G. Bohmer \& N. Chan: arxiv:1409.5585 (2014). 2

[46] R.G. Salcedo, et al.: Eur. J. Phys. 32, 025008 (2015). 2

[47] N. Roy \& N. Banerjee: Euro. Phys. J. Plus, 129, 162 (2014). 2

[48] N. Roy \& N. Banerjee: Annals Phys. 356, 452-466 (2015). 2

[49] P. Rudra, R. Biswas \& U. Debnath: Astrophys. Space Sci. 339, 54 (2012). 2

[50] C.G. Bohmer, G.C. Cabral, N. Chan, R. Lazkoz \& R. Maartens: Phys. Rev. D, 81, 083003 (2010). 2, 26

[51] C.G. Bohmer \& T. Harko: J. Nonlinear Math. Phys. 17, 503 (2010). 2

[52] A.M.V. Toribio: Braz. J. Phys. 36, 772 (2006). 2

[53] N. Tamanini: Phys. Rev. D 89, 083521 (2014). 2

[54] G. Kofinas, E. Papantonopoulos \& E.N. Saridakis: arxiv: 1602.02687 (2016). 2

[55] V. Faraoni \& C.S. Protheroe: arxiv:1209.3726 (2012). 2 
[56] S. Nojiri, S.D. Odintsov, V.K. Olkonomou \& E.N. Saridakis: J. Cosmol. Astropart. Phys. 09, 044 (2015). 2

[57] E. J. Copeland, A.R. Liddle \& D. Wands: Phys. Rev. D, 57, 4686 (1998). 2

[58] A. Paliathanasis, et al: arxiv:1503.05750 (2015). 2

[59] J.F. Jesus, J.L.G. Malatrasi \& S.H. Pereira: arxiv:1504.04037 (2015). 2

[60] S. Carloni, et al.: arxiv: 1512.06996 (2015). 2

[61] R.M. Avagyam, E.V. Chubaryan, G.H. Harutyunyan \& A.A. Saharian: arxiv: 1602.00685 (2016). 2

[62] S.K. Biswas \& S. Chakraborty: Int. J. Mod. Phys. D. (accepted) arxiv: 1504.002431 (2015). 2

[63] J. Dutta \& H. Zonunmawia: arxiv: 1601.00283 (2016). 2

[64] M. Gosenca \& P. Coles: arxiv: 1502.04020 (2015). 2

[65] R. Chaubey \& Rakesh Raushan: Astrophys. Space Sci. 361 (7) 1-9 (2016). 2

[66] R. Chaubey \& Rakesh Raushan: Int. J. Geom. Meth. Mod. Phys. 13 (10) 1650123 (2016). 2 